\title{
Unified formulation applied to free vibrations finite element analysis of beams with arbitrary section
}

\author{
E. Carrera*, M. Petrolo and P. Nali \\ Department of Aeronautics and Space Engineering, Politecnico di Torino, Torino, Italy
}

Received 24 March 2009

Revised 7 October 2009

\begin{abstract}
This paper presents hierarchical finite elements on the basis of the Carrera Unified Formulation for free vibrations analysis of beam with arbitrary section geometries. The displacement components are expanded in terms of the section coordinates, $(\mathrm{x}, \mathrm{y})$, using a set of 1-D generalized displacement variables. N-order Taylor type expansions are employed. $\mathrm{N}$ is a free parameter of the formulation, it is supposed to be as high as 4 . Linear ( 2 nodes), quadratic ( 3 nodes) and cubic (4 nodes) approximations along the beam axis, (z), are introduced to develop finite element matrices. These are obtained in terms of a few fundamental nuclei whose form is independent of both $\mathrm{N}$ and the number of element nodes. Natural frequencies and vibration modes are computed. Convergence and assessment with available results is first made considering different type of beam elements and expansion orders. Additional analyses consider different beam sections (square, annular and airfoil shaped) as well as boundary conditions (simply supported and cantilever beams). It has mainly been concluded that the proposed model is capable of detecting 3-D effects on the vibration modes as well as predicting shell-type vibration modes in case of thin walled beam sections.
\end{abstract}

\section{Introduction}

Beam structures are widely used in many engineering applications. Well-known examples are aircraft wings and helicopter rotor blades in aerospace engineering, and concrete made beams in civil constructions. Classical 1-D models for beams made of isotropic materials are based on the Euler-Bernoulli and Timoshenko theories. The former does not account for transverse shear effects on cross-sections deformations. The latter provides a model which foresees a constant shear deformation distribution on the cross-sections. Both models yield better results for slender beams than for short beams and do not properly account for bending/torsion coupling. Higher-order beam elements are required in engineering fields as aeroelasticity where the proper analysis of torsional and bending vibration modes is fundamental to predict aeroelastic responses as well as critical phenomena such as flutter.

A review of several beam and plate theories for vibration, wave propagations, buckling and post-buckling has been presented by Kapania and Raciti [1,2]. Particular attention was given to models that account for transverse shear-deformation. A review about developments in finite element formulations for thin and thick laminated beams was provided.

Eisenberger et al. [3] have presented a method to compute the exact vibration frequencies of asymmetrical laminated beams. Shear deformations and rotary inertia effects were accounted for. General layouts and geometries of the structure can be analyzed using this method.

* Corresponding author: E. Carrera, professor of Aerospace Structures and Aeroelasticity, Department of Aeronautic and Space Engineering, Politecnico di Torino, Corso Duca degli Abruzzi 24, 10129 Torino, Italy. Tel.: +39 11090 6836; Fax: +39 110906899 ; E-mail: erasmo.carrera@ polito.it. 
Marur and Kant [4] have proposed three higher order models to analyze free vibrations of deep fiber reinforced composite beams. Across the cross-section, axial and shear strain variations were assumed to be cubic and quadratic, respectively. These models accounted for warping of cross-section and shear correction coefficient was not required.

Recently, the same authors [5] have used higher order theory and isoparametric 1-D finite element formulations to study vibrations of angle ply laminated beams. Transverse shear and normal strain effects were accounted for. Symmetric and asymmetric, deep and thin, angle ply beam were investigated.

Kant et al. [6] have formulated an analytical solution for natural frequencies of composite and sandwich beams based on a higher order refined theory. Their model accounted for cubic axial, transverse shear and quadratic transverse normal strain. Numerical experiments showed that the higher-order model furnish lower frequencies, moreover the first-order model is influenced by shear correction factor.

Shi and Lam [7] have conducted free vibrations analysis of composite beam using a finite element formulation based on a third-order shear deformation beam theory. Their studies on flexural frequencies showed that the influence of higher-order terms is negligible on the fundamental frequencies, while it is significant on the frequencies of high flexural modes.

Zhou and Cheung [8] have addressed the problem of transverse vibrations of tapered beams with continuously varying rectangular cross-section. The equation of motion were obtained exploiting Euler-Bernoulli model, eigenfrequency equation was developed by the Rayleigh-Ritz method.

A closed form solution with higher-order mixed theory for free vibrations analysis of composite beams has been presented by Kameswara et al. [9]. Numerical examples were conducted on beams of various span-to-height ratios. The results showed that the presented theory offers significantly lower natural frequencies than those computed through Timoshenko model in case of thick sandwich beams.

Lee and Wooh [10] have developed a technique based on higher-order plate theory to analyze static and free vibrations behavior of box beam composite structures. Their work underlined the importance of shear deformation in improving the accuracy of the free vibrations analysis of composite beams.

A higher-order finite element model based on classical laminated theory has been developed by Ganesan and Zabihollah [11,12]. Vibration response of laminated tapered composite beams was investigated. Curvature was a degree of freedom of each element. This feature ensured continuous curvature and stress distribution across element interfaces. Higher frequencies analysis capabilities were then enhanced. It was also shown how a higher-order formulation needs fewer elements to obtain accurate results.

Şimşek and Kocatürk [13] have used a third-order shear deformation theory to study free vibrations of beams with different boundary conditions. Results were compared with the Timoskenko and Euler-Bernoulli models responses. Investigating different thickness-to-length ratios, it was highlighted that higher-order models furnish significantly better results than classical theories in case of short beams and high mode numbers.

Hsu et alii [14] have dealt with free-vibrations analysis of non-uniform Euler-Bernoulli beam exploiting the Adomian modified decomposition method. Various boundary conditions were accounted for. The efficiency of the method was shown comparing the results with those furnished in literature.

This paper presents the free vibrations finite element analysis of beams based on higher-order models. The proposed formulation is embedded in the framework of the Carrera Unified Formulation (CUF) [15,16]. CUF offers a systematic procedure to obtain refined structural models considering the order of the theory as a free parameter of the formulation. Different beam elements (2,3, and 4 nodes) as well as different higher-order models for cross-section displacements field are used. Their development and static analysis assessments have been recently presented in Carrera et al. [17]. Euler-Bernoulli and Timoshenko beam models are obtained as particular cases of the first-order formulation. Homogenous cross-sections and isotropic materials are considered. Three main problems are analyzed: a rectangular simply supported cross shaped beam, an annular cross shaped beam, and a wing/rotor blade model. Natural frequencies and modal shapes are computed. The results are compared with benchmarks retrieved from the classical theories and with shell finite element and analytical models.

\section{Preliminaries}

The adopted coordinate frame is presented in Fig. 1. The beam boundaries over $z$ are $0 \leqslant z \leqslant L$. The origin is placed on the center of gravity of the section even when a generic not-rectangular shape is considered. The 


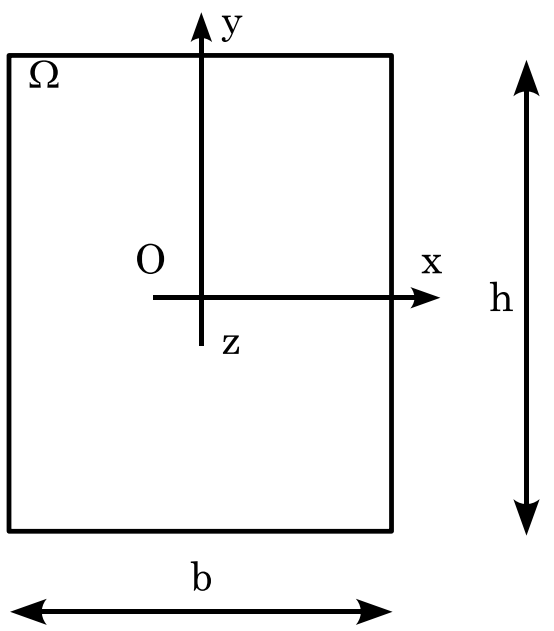

Fig. 1. Coordinate frame and geometry of a rectangular cross-section.

displacements vector is:

$$
\mathbf{u}(x, y, z)=\left\{u_{x} u_{y} u_{z}\right\}^{T}
$$

Superscript T represents the transposition operator. The stress, $\sigma$, and the strain, $\epsilon$, are grouped as it follows:

$$
\begin{aligned}
& \boldsymbol{\sigma}_{p}=\left\{\sigma_{x x} \sigma_{y y} \sigma_{x y}\right\}^{T}, \boldsymbol{\epsilon}_{p}=\left\{\epsilon_{x x} \epsilon_{y y} \epsilon_{x y}\right\}^{T} \\
& \boldsymbol{\sigma}_{n}=\left\{\sigma_{x z} \sigma_{y z} \sigma_{z z}\right\}^{T}, \boldsymbol{\epsilon}_{n}=\left\{\epsilon_{x z} \epsilon_{y z} \epsilon_{z z}\right\}^{T}
\end{aligned}
$$

Subscript " $n$ " stands for terms laying on the cross-section, while " $p$ " stands for terms laying on planes orthogonal to $\Omega$. Linear strain-displacement relations are used:

$$
\begin{aligned}
& \boldsymbol{\epsilon}_{p}=\boldsymbol{D}_{p} \mathbf{u} \\
& \boldsymbol{\epsilon}_{n}=\boldsymbol{D}_{n} \mathbf{u}=\left(\boldsymbol{D}_{n \Omega}+\boldsymbol{D}_{n z}\right) \mathbf{u}
\end{aligned}
$$

with:

$$
\boldsymbol{D}_{p}=\left[\begin{array}{ccc}
\frac{\partial}{\partial x} & 0 & 0 \\
0 & \frac{\partial}{\partial y} & 0 \\
\frac{\partial}{\partial y} & \frac{\partial}{\partial x} & 0
\end{array}\right], \boldsymbol{D}_{n \Omega}=\left[\begin{array}{ccc}
0 & 0 & \frac{\partial}{\partial x} \\
0 & 0 & \frac{\partial}{\partial y} \\
0 & 0 & 0
\end{array}\right], \boldsymbol{D}_{n z}=\left[\begin{array}{ccc}
\frac{\partial}{\partial z} & 0 & 0 \\
0 & \frac{\partial}{\partial z} & 0 \\
0 & 0 & \frac{\partial}{\partial z}
\end{array}\right]
$$

In case of orthotropic materials, the Hooke law holds:

$$
\sigma=C \epsilon
$$

According to Eq (2), the previous equation becomes:

$$
\begin{aligned}
\boldsymbol{\sigma}_{p} & =\tilde{\boldsymbol{C}}_{p p} \boldsymbol{\epsilon}_{p}+\tilde{\boldsymbol{C}}_{p n} \boldsymbol{\epsilon}_{n} \\
\boldsymbol{\sigma}_{n} & =\tilde{\boldsymbol{C}}_{n p} \boldsymbol{\epsilon}_{p}+\tilde{\boldsymbol{C}}_{n n} \boldsymbol{\epsilon}_{n}
\end{aligned}
$$

where matrices $\tilde{\boldsymbol{C}}_{p p}, \tilde{\boldsymbol{C}}_{n n}, \tilde{\boldsymbol{C}}_{p n}$ and $\tilde{\boldsymbol{C}}_{n p}$ are:

$$
\tilde{\boldsymbol{C}}_{p p}=\left[\begin{array}{lll}
\tilde{C}_{11} & \tilde{C}_{12} & \tilde{C}_{16} \\
\tilde{C}_{12} & \tilde{C}_{22} & \tilde{C}_{26} \\
\tilde{C}_{16} & \tilde{C}_{26} & \tilde{C}_{66}
\end{array}\right], \quad \tilde{\boldsymbol{C}}_{n n}=\left[\begin{array}{ccc}
\tilde{C}_{55} & \tilde{C}_{45} & 0 \\
\tilde{C}_{45} & \tilde{C}_{44} & 0 \\
0 & 0 & \tilde{C}_{33}
\end{array}\right], \quad \tilde{\boldsymbol{C}}_{p n}=\tilde{\boldsymbol{C}}_{n p}^{T}=\left[\begin{array}{lll}
0 & 0 & \tilde{C}_{13} \\
0 & 0 & \tilde{C}_{23} \\
0 & 0 & \tilde{C}_{36}
\end{array}\right]
$$

For the sake of brevity, the dependence of the coefficients $[\tilde{C}]_{i j}$ versus Young's moduli, Poisson's ratio, the shear moduli and the fibre angle is not reported. It can be found in Tsai [18] or Reddy [19]. 
Table 1

Mac Laurin's polynomials

\begin{tabular}{ccl}
\hline 0 & 1 & $F_{1}=1$ \\
\hline 1 & 3 & $F_{2}=x \quad F_{3}=y$ \\
2 & 6 & $F_{4}=x^{2} \quad F_{5}=x y \quad F_{6}=y^{2}$ \\
3 & 10 & $F_{7}=x^{3} \quad F_{8}=x^{2} y \quad F_{9}=x y^{2} \quad F_{10}=y^{3}$ \\
$\ldots$ & $\ldots$ & $\ldots$ \\
$N$ & $\frac{(N+1)(N+2)}{2}$ & $F_{\left(N^{2}+N+2\right)}=x^{N} F_{\frac{\left(N^{2}+N+4\right)}{2}}=x^{N-1} y \quad \ldots \quad F_{\frac{N(N+3)}{2}}=x y^{N-1} F_{\frac{(N+1)(N+2)}{2}}=y^{N}$ \\
\hline
\end{tabular}

\section{Unified formulation}

In the framework of the Carrera Unified Formulation (CUF) [15], the displacement field is assumed as an expansion of a certain class of functions $F_{\tau}$ :

$$
\mathbf{u}=F_{\tau} \mathbf{u}_{\tau}, \quad \tau=1,2, \ldots, N_{u}
$$

where $F_{\tau}$ are functions of the coordinates $x$ and $y$ on the cross-section. $\mathbf{u}_{\tau}$ is the displacement vector and $N_{u}$ stands for the number of terms of the expansion. According to the Einstein notation, the repeated subscript $\tau$ indicates summation. Mac Laurin polynomials are chosen as expansion functions. The maximum expansion order, $N$, is supposed to be 4 . Table 1 presents $N_{u}$ and $F_{\tau}$ as functions of $N$. For example, the second-order displacement field is:

$$
\begin{aligned}
& u_{x}=u_{x 1}+x u_{x 2}+y u_{x 3}+x^{2} u_{x 4}+x y u_{x 5}+y^{2} u_{x 6} \\
& u_{y}=u_{y 1}+x u_{y 2}+y u_{y 3}+x^{2} u_{y 4}+x y u_{y 5}+y^{2} u_{y 6} \\
& u_{z}=u_{z 1}+x u_{z 2}+y u_{z 3}+x^{2} u_{z 4}+x y u_{z 5}+y^{2} u_{z 6}
\end{aligned}
$$

The Timoshenko beam model (TBM) can be obtained by acting on $F_{\tau}$ expansion. Two conditions have to be imposed. 1) first-order approximation kinematic field:

$$
\begin{aligned}
& u_{x}=u_{x 1}+x u_{x 2}+y u_{x 3} \\
& u_{y}=u_{y 1}+x u_{y 2}+y u_{y 3} \\
& u_{z}=u_{z 1}+x u_{z 2}+y u_{z 3}
\end{aligned}
$$

2) the displacement components $u_{x}$ and $u_{y}$ have to be constant above the cross-section:

$$
u_{x 2}=u_{y 2}=u_{x 3}=u_{y 3}=0
$$

The Euler-Bernoulli beam (EBBM) can also be obtained through penalization of $\epsilon_{x z}$ and $\epsilon_{y z}$. This condition can be done by using a penalty value $\chi$ in the following constitutive equations:

$$
\begin{aligned}
& \sigma_{x z}=\chi \tilde{C}_{55} \epsilon_{x z}+\chi \tilde{C}_{45} \epsilon_{y z} \\
& \sigma_{y z}=\chi \tilde{C}_{45} \epsilon_{x z}+\chi \tilde{C}_{44} \epsilon_{y z}
\end{aligned}
$$

Classical theories and first-order models require the assumption of opportunely reduced material stiffness coefficients to correct Poisson's locking (see Carrera and Brischetto [20,21]). Unless differently specified, for classical and first-order models Poisson's locking is corrected according to Carrera and Giunta [22].

\section{Finite element formulation}

By introducing the shape functions, $N_{i}$, and the nodal displacement vector, $\mathbf{q}_{\tau i}$ :

$$
\mathbf{q}_{\tau i}=\left\{q_{u_{x_{\tau i}}} q_{u_{y_{\tau i}}} q_{u_{z_{\tau i}}}\right\}^{T}
$$

the displacement vector becomes:

$$
\mathbf{u}_{\tau}=N_{i} F_{\tau} \mathbf{q}_{\tau i}
$$

For the sake of brevity, the shape functions are not reported here. They can be found in Bathe [23]. Elements with 2, 3 and 4 nodes are formulated. These elements are addressed as B2, B3, B4, respectively. The stiffness and mass 
matrices of the elements and the external loadings that are coherent to the models are obtained via the Principle of Virtual Displacements:

$$
\delta L_{i n t}=\int_{V}\left(\delta \boldsymbol{\epsilon}_{p}^{T} \boldsymbol{\sigma}_{p}+\delta \boldsymbol{\epsilon}_{n}^{T} \boldsymbol{\sigma}_{n}\right) d V=\delta L_{e x t}-\delta L_{\text {ine }}
$$

where $L_{\text {int }}$ stands for the strain energy, $L_{\text {ext }}$ is the work of the external loadings, and $L_{\text {ine }}$ is the work of the inertial loadings. $\delta$ stands for the virtual variation. The virtual variation of the strain energy is rewritten using Eqs (3), (6) and (14):

$$
\begin{aligned}
\delta L_{i n t}= & \int_{l} \delta \mathbf{q}_{\tau i}^{T} N_{i}\left[\int_{\Omega} \mathbf{D}_{p}^{T}\left(F_{\tau} \mathbf{I}\right) \tilde{\boldsymbol{C}}_{p p} \mathbf{D}_{p}\left(F_{s} \mathbf{I}\right) d \Omega\right] N_{j} \mathbf{q}_{s j} d z+ \\
& +\int_{l} \delta \mathbf{q}_{\tau i}^{T} N_{i}\left[\int_{\Omega} \mathbf{D}_{p}^{T}\left(F_{\tau} \mathbf{I}\right) \tilde{\boldsymbol{C}}_{p n} \mathbf{D}_{n \Omega}\left(F_{s} \mathbf{I}\right) d \Omega\right] N_{j} \mathbf{q}_{s j} d z+ \\
& +\int_{l} \delta \mathbf{q}_{\tau i}^{T} N_{i}\left[\int_{\Omega} \mathbf{D}_{p}^{T}\left(F_{\tau} \mathbf{I}\right) \tilde{\boldsymbol{C}}_{p n} F_{s} d \Omega\right] N_{j, z} \mathbf{q}_{s j} d z+ \\
& +\int_{l} \delta \mathbf{q}_{\tau i}^{T} N_{i}\left[\int_{\Omega} \mathbf{D}_{n \Omega}^{T}\left(F_{\tau} \mathbf{I}\right) \tilde{\boldsymbol{C}}_{n p} \mathbf{D}_{p}\left(F_{s} \mathbf{I}\right) d \Omega\right] N_{j} \mathbf{q}_{s j} d z+ \\
& +\int_{l} \delta \mathbf{q}_{\tau i}^{T} N_{i}\left[\int_{\Omega} \mathbf{D}_{n \Omega}^{T}\left(F_{\tau} \mathbf{I}\right) \tilde{\boldsymbol{C}}_{n n} \mathbf{D}_{n \Omega}\left(F_{s} \mathbf{I}\right) d \Omega\right] N_{j} \mathbf{q}_{s j} d z+ \\
& +\int_{l} \delta \mathbf{q}_{\tau i}^{T} N_{i}\left[\int_{\Omega} \mathbf{D}_{p}^{T}\left(F_{\tau} \mathbf{I}\right) \tilde{\boldsymbol{C}}_{n n} F_{s} d \Omega\right] N_{j, z} \mathbf{q}_{s j} d z+ \\
& +\int_{l} \delta \mathbf{q}_{\tau i}^{T} N_{i_{z}}\left[\int_{\Omega} F_{\tau} \tilde{\boldsymbol{C}}_{n p} \mathbf{D}_{p}\left(F_{s} \mathbf{I}\right) d \Omega\right] N_{j} \mathbf{q}_{s j} d z+ \\
& +\int_{l} \delta \mathbf{q}_{\tau i}^{T} N_{i_{,}}\left[\int_{\Omega} F_{\tau} \tilde{\boldsymbol{C}}_{n n} \mathbf{D}_{n \Omega}\left(F_{s} \mathbf{I}\right) d \Omega\right] N_{j} \mathbf{q}_{s j} d z+ \\
& +\int_{l} \delta \mathbf{q}_{\tau i}^{T} N_{i_{z}}\left[\int_{\Omega} F_{\tau} \tilde{\boldsymbol{C}}_{n n} F_{s} d \Omega\right] N_{j, z} \mathbf{q}_{s j} d z
\end{aligned}
$$

where $\mathbf{I}$ is the identity matrix. Eq. (16) in a compact format becomes:

$$
\delta L_{i n t}=\delta \mathbf{q}_{\tau i}^{T} \mathbf{K}^{i j \tau s} \mathbf{q}_{s j}
$$

where $\mathbf{K}^{i j \tau s}$ is the stiffness matrix in the form of the fundamental nucleus.

In order to simplify the expressions of the components of $\mathbf{K}^{i j \tau s}$, the line and surface integrals are indicated by letter $I$ whose subscripts point the integration domain and superscripts point either $N_{i}$ or $F_{\tau}$ and their derivatives. For example two integrals and their respective notations are shown:

$$
I_{\Omega}^{\tau, x} s, y=\int_{\Omega} F_{\tau, x} F_{s, y} d \Omega, \quad F_{\tau, x}=\frac{\partial F_{\tau}}{\partial x}, \quad F_{s, y}=\frac{\partial F_{s}}{\partial y}
$$

and:

$$
I_{l}^{i, z j, z}=\int_{l} N_{i, z} N_{j, z} d z, \quad N_{i, z}=\frac{\partial N_{i}}{\partial z}, \quad N_{j, z}=\frac{\partial N_{j}}{\partial z}
$$

Substituting Eqs (18) and (19) in Eq. (17), a compact form of the fundamental nucleus components is obtained: 


$$
\begin{aligned}
& K_{x x}^{i j \tau s}=\tilde{C}_{11} I_{\Omega}^{\tau, x}{ }^{s, x} I_{l}^{i j}+\tilde{C}_{16} I_{\Omega}^{\tau, y}{ }^{,,_{x}} I_{l}^{i j}+\tilde{C}_{16} I_{\Omega}^{\tau, x}{ }^{,_{y}} I_{l}^{i j}+\tilde{C}_{66} I_{\Omega}^{\tau, y s}{ }^{,_{y}} I_{l}^{i j}+\tilde{C}_{55} I_{\Omega}^{\tau s} I_{l}^{i, z j, z} \\
& K_{x y}^{i j \tau s}=\tilde{C}_{12} I_{\Omega}^{\tau,{ }_{x} s, y} I_{l}^{i j}+\tilde{C}_{26} I_{\Omega}^{\tau, y}{ }^{s, y} I_{l}^{i j}+\tilde{C}_{16} I_{\Omega}^{\tau,{ }_{x}{ }^{s, x}} I_{l}^{i j}+\tilde{C}_{66} I_{\Omega}^{\tau,{ }_{y} s,_{x}} I_{l}^{i j}+\tilde{C}_{45} I_{\Omega}^{\tau s} I_{l}^{i,{ }_{z} j, z} \\
& K_{x z}^{i j \tau s}=\tilde{C}_{13} I_{\Omega}^{\tau, x} s I_{l}^{i j, z}+\tilde{C}_{36} I_{\Omega}^{\tau, y} s I_{l}^{i j, z}+\tilde{C}_{55} I_{\Omega}^{\tau s,{ }_{x}} I_{l}^{i, z} j+\tilde{C}_{45} I_{\Omega}^{\tau s, y} I_{l}^{i, z j}
\end{aligned}
$$

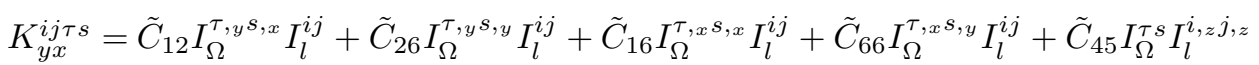

$$
\begin{aligned}
& K_{y y}^{i j \tau s}=\tilde{C}_{22} I_{\Omega}^{\tau, y}{ }^{,{ }_{y}} I_{l}^{i j}+\tilde{C}_{26} I_{\Omega}^{\tau, x} s^{, y} I_{l}^{i j}+\tilde{C}_{26} I_{\Omega}^{\tau, y}{ }^{,_{x}} I_{l}^{i j}+\tilde{C}_{66} I_{\Omega}^{\tau, x}{ }^{,_{x}} I_{l}^{i j}+\tilde{C}_{44} I_{\Omega}^{\tau s} I_{l}^{i, z j, z} \\
& K_{y z}^{i j \tau s}=\tilde{C}_{23} I_{\Omega}^{\tau, y s} I_{l}^{i j, z}+\tilde{C}_{36} I_{\Omega}^{\tau,{ }_{x} s} I_{l}^{i j,,_{z}}+\tilde{C}_{45} I_{\Omega}^{\tau s,{ }_{x}} I_{l}^{i,,_{z} j}+\tilde{C}_{44} I_{\Omega}^{\tau s,{ }_{y}} I_{l}^{i,,_{z} j} \\
& K_{z x}^{i j \tau s}=\tilde{C}_{55} I_{\Omega}^{\tau, x} s I_{l}^{i j, z}+\tilde{C}_{45} I_{\Omega}^{\tau, y} I_{l}^{i j, z}+\tilde{C}_{13} I_{\Omega}^{\tau s, x} I_{l}^{i, z j}+\tilde{C}_{36} I_{\Omega}^{\tau s, y} I_{l}^{i, z j} \\
& K_{z y}^{i j \tau s}=\tilde{C}_{45} I_{\Omega}^{\tau,{ }_{x} s} I_{l}^{i j,{ }_{z}}+\tilde{C}_{44} I_{\Omega}^{\tau, y} s I_{l}^{i j,_{z}}+\tilde{C}_{23} I_{\Omega}^{\tau s,{ }_{y}} I_{l}^{i,{ }_{z} j}+\tilde{C}_{36} I_{\Omega}^{\tau s,{ }_{x}} I_{l}^{i,{ }_{z} j} \\
& K_{z z}^{i j \tau s}=\tilde{C}_{55} I_{\Omega}^{\tau,{ }_{x}{ }^{s}{ }_{x}} I_{l}^{i j}+\tilde{C}_{45} I_{\Omega}^{\tau,{ }_{y} s,{ }_{x}} I_{l}^{i j}+\tilde{C}_{45} I_{\Omega}^{\tau,{ }_{x} s_{y}} I_{l}^{i j}+\tilde{C}_{44} I_{\Omega}^{\tau,{ }_{y} s,_{y}} I_{l}^{i j}+\tilde{C}_{33} I_{\Omega}^{\tau s} I_{l}^{i,{ }_{z} j, z}
\end{aligned}
$$

Line integrals of shape functions are numerically computed via Gauss' method. Selective integration is adopted to overcome shear locking phenomena (see Bathe [23]). Surface integrals of $F_{\tau}$ are evaluated numerically by partitioning the integration area in a certain number of sub-domains:

$$
\int_{\Omega} f(x, y) d \Omega \simeq \sum_{m=1}^{M} f\left(x_{m}, y_{m}\right) \Omega_{m}
$$

where $x_{m}$ and $y_{m}$ are the coordinates of the center of gravity of $\Omega_{m} . M$ is evaluated through a convergence study. This numerical technique is adopted to be able to analyze arbitrary cross-section geometries since analytical solutions of the surface integrals could not be efficient from a numerical point of view. The sub-domains can have arbitrary geometries. The triangular shape is usually preferred since it permits a better partitioning of irregular cross-sections (e.g. annular or airfoil shaped).

The virtual variation of the work of the inertial loadings is:

$$
\delta L_{i n e}=\int_{V} \rho \ddot{\boldsymbol{u}} \delta \boldsymbol{u}^{T} d V
$$

where $\rho$ stands for the density of the material, and $\ddot{\boldsymbol{u}}$ is the acceleration vector. Equation (22) is rewritten using Eqs (3), and (14):

$$
\delta L_{i n e}=\int_{l} \delta \mathbf{q}_{\tau i}^{T} N_{i}\left[\int_{\Omega} \rho\left(F_{\tau} \mathbf{I}\right)\left(F_{s} \mathbf{I}\right) d \Omega\right] N_{j} \ddot{\boldsymbol{q}}_{s j} d z
$$

where $\ddot{\boldsymbol{q}}$ is the nodal acceleration vector. The last equation can be rewritten in the following compact manner:

$$
\delta L_{i n e}=\delta \mathbf{q}_{\tau i}^{T} \mathbf{M}^{i j \tau s} \ddot{\boldsymbol{q}}_{s j}
$$

where $\mathbf{M}^{i j \tau s}$ is the mass matrix in the form of the fundamental nucleus. Its components are:

$$
\begin{gathered}
M_{x x}^{i j \tau s}=M_{y y}^{i j \tau s}=M_{z z}^{i j \tau s}=\rho I_{\Omega}^{\tau s} I_{l}^{i j} \\
M_{x y}^{i j \tau s}=M_{x z}^{i j \tau s}=M_{y x}^{i j \tau s}=M_{y z}^{i j \tau s}=M_{z x}^{i j \tau s}=M_{z y}^{i j \tau s}=0
\end{gathered}
$$

It should be noted that no assumptions on the approximation order have been done. It is therefore possible to obtain refined beam models without changing the formal expression of the nucleus components of the stiffness matrix, $\mathbf{K}$, and of the mass matrix, M. For the sake of brevity, the derivation of the loadings vector variationally coherent to the model is not reported, it can be found in Carrera et al. [17]. The undamped dynamic problem can be written as it follows: 


$$
M \ddot{a}+K[\boldsymbol{a}]=\boldsymbol{p}
$$

where $\boldsymbol{a}$ is the vector of the nodal unknowns and $\boldsymbol{p}$ is the loadings vector. Introducing harmonic solutions, it is possible to compute the natural frequencies, $\omega_{k}$, for the homogenous case, by solving an eigenvalues problem:

$$
\left(-\omega_{k}^{2} \mathbf{M}+\mathbf{K}\right) \boldsymbol{a}_{k}=0
$$

where $\boldsymbol{a}_{k}$ is the $k$-th eigenvector.

\section{Results and discussion}

Free vibrations of different beam models are analyzed. Clamped and simply supported boundary conditions are accounted for. The beams are supposed to have square cross-section, annular cross-section or an airfoil shaped section. An isotropic material is used. Young's modulus, $E$, is equal to 75 [GPa]. The Poisson ratio, $\nu$, is equal to 0.33 .

\subsection{Square cross-section beam}

A simply supported beam is considered as a first assessment of the proposed FEs. The coordinate frame and the cross-section geometry are shown in Fig. 1 . The sides of the section are equal to 0.2 [m]. The span-to-height ratio, $L / h$, is equal to 100 and 10 . Slender and relatively short beams are therefore investigated. The first five bending modes are analyzed. As benchmark for the $k$-th natural frequency, Euler-Bernoulli's solution for simply supported beams [24] is used:

$$
f_{k_{b}}=\frac{\pi}{2}\left(\frac{k}{L}\right)^{2}\left(\frac{E I}{\rho A}\right)^{\frac{1}{2}}
$$

The first five frequencies of the beam are reported in Tables 2, 3, 4 and 5 . They have been computed for different beam elements, expansion orders and span-to-height ratios. Considering slender beams, the results match the benchmark values. In case of short beams, the differences between the FE and the benchmark solution become remarkable as the frequencies grow. As far as the first natural frequency of the slender beams is concerned, no differences have been found for $N>2$. Ten B3 elements are enough to get the convergence. For higher frequencies a third-order approximation and $40 \mathrm{~B} 3$ elements are needed. Short beams require fourth-order models as well as a higher number of elements. In both cases, first-order approximation matches Timoshenko's model. Linear terms of $u_{x}$ and $u_{y}$ can be neglected. Higher-order than linear approximation yields a more flexible structure. This effect is not found in case of linear expansion because of the presence of Poisson's locking correction effect. A higher number of elements enhances the flexibility of the structure. In Fig. 2 two bending modes are shown underlining the 3-D capabilities of the adopted finite element formulation.

\subsection{Annular cross-section beam}

A beam with annular cross-section is considered. The coordinate frame and the cross-section geometry are shown in Fig. 3. The outer diameter of the section is equal to $2[\mathrm{~m}]$. While the inner diameter is equal to $1.96[\mathrm{~m}]$. The span-to-diameter ratio, $L / d$, is equal to 100 and 10 .

In Table 6 the solution obtained through the present beam model is compared to that furnished by NASTRAN using shell elements. The beam is clamped. Bending and radial modes are considered. In both cases the beam model is able to match the shell solution. In Figs 4 and 5 the second radial mode is plotted as it is computed by NASTRAN and CUF beam model. A further comparison is addressed in Table 7 where a Navier type closed form solution for shell structures is adopted as in [25-27]. The beam is simply supported. Bending and circumferential modes are accounted for. The beam model solution matches the analytical one.

In Table 8 the first five natural frequencies related to bending modes are shown. They have been computed for different expansion orders and span-to-height ratios, $40 \mathrm{~B} 4$ elements have been used. The beam is simply supported. Reference solutions for the bending modes frequencies are obtained through Eq. (28). The positions held by each 
Table 2

Effect of the number of elements on $f_{1},[\mathrm{~Hz}]$. Square cross-section beam. $L / h=100$

\begin{tabular}{cccccc}
\hline No. Elem. & Elem. Type & EBBM & TBM & $N=1$ & $N=2$ \\
\hline \multicolumn{5}{c}{$f_{1_{b}}=1.195[\mathrm{~Hz}]$} \\
10 & B2 & 1.209 & 1.209 & 1.209 & 1.212 \\
20 & B2 & 1.198 & 1.198 & 1.198 & 1.199 \\
40 & B2 & 1.195 & 1.195 & 1.195 & 1.195 \\
10 & B3 & 1.195 & 1.194 & 1.194 & 1.194 \\
10 & B4 & 1.195 & 1.194 & 1.194 & 1.194 \\
\hline
\end{tabular}

Table 3

Effect of the number of elements on $f_{2}, f_{3}, f_{4}$ and $f_{5},[\mathrm{~Hz}]$. Square cross-section beam. $L / h=100$

\begin{tabular}{|c|c|c|c|c|c|c|}
\hline No. Elem. & Elem. Type & EBBM & TBM & $N=1$ & $N=2$ & $N=3$ \\
\hline \multicolumn{7}{|c|}{$f_{2_{b}}=4.780[\mathrm{~Hz}]$} \\
\hline 10 & B2 & 5.023 & 5.020 & 5.020 & 5.061 & 5.061 \\
\hline 20 & B2 & 4.837 & 4.835 & 4.834 & 4.845 & 4.844 \\
\hline 40 & B2 & 4.792 & 4.790 & 4.790 & 4.793 & 4.792 \\
\hline 10 & B3 & 4.779 & 4.777 & 4.777 & 4.777 & 4.777 \\
\hline 20 & B3 & 4.778 & 4.776 & 4.778 & 4.776 & 4.775 \\
\hline 40 & B3 & 4.778 & 4.775 & 4.775 & 4.775 & 4.775 \\
\hline 10 & B4 & 4.778 & 4.775 & 4.775 & 4.775 & 4.775 \\
\hline \multicolumn{7}{|c|}{$f_{3_{b}}=10.755[\mathrm{~Hz}]$} \\
\hline 10 & B2 & 12.053 & 12.039 & 12.039 & 12.268 & 12.265 \\
\hline 20 & B2 & 11.052 & 11.041 & 11.041 & 11.091 & 11.089 \\
\hline 40 & B2 & 10.822 & 10.811 & 10.811 & 10.824 & 10.822 \\
\hline 10 & B3 & 10.764 & 10.753 & 10.753 & 10.754 & 10.752 \\
\hline 20 & B3 & 10.748 & 10.738 & 10.738 & 10.738 & 10.736 \\
\hline 40 & B3 & 10.747 & 10.737 & 10.737 & 10.737 & 10.735 \\
\hline 10 & B4 & 10.747 & 10.737 & 10.737 & 10.737 & 10.735 \\
\hline \multicolumn{7}{|c|}{$f_{4_{h}}=19.119[\mathrm{~Hz}]$} \\
\hline 10 & B2 & 23.546 & 23.491 & 23.491 & 24.319 & 24.309 \\
\hline 20 & B2 & 20.080 & 20.043 & 20.043 & 20.206 & 20.200 \\
\hline 40 & B2 & 19.338 & 19.304 & 19.304 & 19.343 & 19.337 \\
\hline 10 & B3 & 19.193 & 19.159 & 19.159 & 19.165 & 19.159 \\
\hline 20 & B3 & 19.107 & 19.073 & 19.073 & 19.074 & 19.069 \\
\hline 40 & B3 & 19.101 & 19.068 & 19.068 & 19.068 & 19.063 \\
\hline 10 & B4 & 19.102 & 19.068 & 19.068 & 19.069 & 19.064 \\
\hline \multicolumn{7}{|c|}{$f_{5_{b}}=29.874[\mathrm{~Hz}]$} \\
\hline 10 & B2 & 41.858 & 41.674 & 41.674 & 44.090 & 44.053 \\
\hline 20 & B2 & 32.278 & 32.180 & 32.180 & 32.596 & 32.579 \\
\hline 40 & B2 & 30.417 & 30.332 & 30.332 & 30.428 & 30.414 \\
\hline 10 & B3 & 30.174 & 30.090 & 30.090 & 30.117 & 30.104 \\
\hline 20 & B3 & 29.857 & 29.775 & 29.775 & 29.777 & 29.764 \\
\hline 40 & B3 & 29.835 & 29.754 & 29.754 & 29.755 & 29.742 \\
\hline 10 & B4 & 29.840 & 29.758 & 29.758 & 29.760 & 29.747 \\
\hline
\end{tabular}

Table 4

Effect of the number of elements on $f_{1}$, [Hz]. Square cross-section beam. $L / h=10$

\begin{tabular}{cccccccc}
\hline No. Elem. & Elem. Type & EBBM & TBM & $N=1$ & $N=2$ & $N=3$ & $N=4$ \\
\hline \multicolumn{7}{c}{$f_{1_{b}}=119.495[\mathrm{~Hz}]$} \\
10 & B2 & 120.442 & 119.139 & 119.139 & 119.398 & 119.189 & 119.188 \\
20 & B2 & 119.334 & 118.058 & 118.058 & 118.142 & 117.938 & 117.938 \\
40 & B2 & 119.059 & 117.790 & 117.790 & 117.831 & 117.629 & 117.628 \\
10 & B3 & 118.970 & 117.704 & 117.704 & 117.730 & 117.528 & 117.528 \\
20 & B3 & 118.968 & 117.701 & 117.701 & 117.728 & 117.526 & 117.525 \\
10 & B4 & 118.968 & 117.701 & 117.701 & 117.728 & 117.526 & 117.525 \\
\hline
\end{tabular}


Table 5

Effect of the number of elements on $f_{2}, f_{3}, f_{4}$ and $f_{5},[\mathrm{~Hz}]$. Square cross-section beam. $L / h=10$

\begin{tabular}{|c|c|c|c|c|c|c|c|}
\hline No. Elem. & Elem. Type & EBBM & TBM & $N=1$ & $N=2$ & $N=3$ & $N=4$ \\
\hline \multicolumn{8}{|c|}{$f_{2_{b}}=477.978[\mathrm{~Hz}]$} \\
\hline 10 & B2 & 493.730 & 473.328 & 473.328 & 477.234 & 474.082 & 474.061 \\
\hline 20 & B2 & 475.873 & 457.071 & 457.071 & 458.270 & 455.421 & 455.402 \\
\hline 40 & B2 & 471.565 & 453.138 & 453.138 & 453.701 & 450.923 & 450.904 \\
\hline 10 & B3 & 470.289 & 451.971 & 451.971 & 452.335 & 449.577 & 449.558 \\
\hline 20 & B3 & 470.152 & 451.846 & 451.846 & 452.203 & 449.447 & 449.428 \\
\hline 40 & B3 & 470.143 & 451.838 & 451.838 & 452.194 & 449.438 & 449.420 \\
\hline 10 & B4 & 470.143 & 451.838 & 451.838 & 452.194 & 449.438 & 449.419 \\
\hline 20 & B4 & 470.143 & 451.838 & 451.838 & 452.193 & 449.438 & 449.419 \\
\hline \multicolumn{8}{|c|}{$f_{3_{b}}=1075.451[\mathrm{~Hz}]$} \\
\hline 10 & B2 & 1156.717 & 1056.451 & 1056.451 & 1074.491 & 1059.816 & 1059.585 \\
\hline 20 & B2 & 1065.371 & 981.051 & 981.051 & 986.227 & 974.316 & 974.136 \\
\hline 40 & B2 & 1044.240 & 963.387 & 963.387 & 965.718 & 954.395 & 954.226 \\
\hline 10 & B3 & 1038.897 & 958.907 & 958.907 & 960.406 & 949.233 & 949.064 \\
\hline 20 & B3 & 1037.438 & 957.684 & 957.684 & 959.105 & 947.969 & 947.802 \\
\hline 40 & B3 & 1037.344 & 957.605 & 957.605 & 959.019 & 947.885 & 947.720 \\
\hline 10 & B4 & 1037.348 & 957.608 & 957.608 & 959.022 & 947.888 & 947.722 \\
\hline 20 & B4 & 1037.338 & 957.600 & 957.600 & 959.013 & 947.879 & 947.713 \\
\hline \multicolumn{8}{|c|}{$f_{4_{b}}=1911.912[\mathrm{~Hz}]$} \\
\hline 10 & B2 & 2172.952 & 1866.073 & 1866.073 & 1916.540 & 1874.242 & 1873.035 \\
\hline 20 & B2 & 1881.179 & 1650.792 & 1650.792 & 1664.375 & 1634.155 & 1633.350 \\
\hline 40 & B2 & 1817.182 & 1602.055 & 1602.055 & 1607.875 & 1580.005 & 1579.276 \\
\hline 10 & B3 & 1804.577 & 1592.389 & 1592.389 & 1596.216 & 1568.820 & 1568.098 \\
\hline 20 & B3 & 1797.092 & 1586.644 & 1586.644 & 1589.041 & 1562.889 & 1562.178 \\
\hline 40 & B3 & 1796.597 & 1586.264 & 1586.264 & 1589.627 & 1562.490 & 1561.784 \\
\hline 10 & B4 & 1796.656 & 1586.309 & 1586.309 & 1589.676 & 1562.537 & 1561.831 \\
\hline 20 & B4 & 1796.565 & 1586.239 & 1586.239 & 1589.600 & 1562.464 & 1561.758 \\
\hline 40 & B4 & 1796.564 & 1586.238 & 1586.238 & 1589.598 & 1562.463 & 1561.757 \\
\hline \multicolumn{8}{|c|}{$f_{5_{b}}=2987.363[\mathrm{~Hz}]$} \\
\hline 10 & B2 & 3630.433 & 2905.289 & 2905.289 & 3010.050 & 2916.029 & 2911.758 \\
\hline 20 & B2 & 2914.789 & 2434.784 & 2434.784 & 2461.974 & 2403.275 & 2400.840 \\
\hline 40 & B2 & 2766.529 & 2331.254 & 2331.254 & 2342.333 & 2289.951 & 2287.836 \\
\hline 10 & B3 & 2747.013 & 2317.445 & 2317.445 & 2325.157 & 2273.664 & 2271.579 \\
\hline 20 & B3 & 2721.350 & 2299.292 & 2299.292 & 2305.461 & 2254.936 & 2252.901 \\
\hline 40 & B3 & 2719.614 & 2298.060 & 2298.060 & 2304.121 & 2253.658 & 2251.638 \\
\hline 10 & B4 & 2719.990 & 2298.327 & 2298.327 & 2304.409 & 2253.932 & 2251.911 \\
\hline 20 & B4 & 2719.504 & 2297.982 & 2297.982 & 2304.033 & 2253.574 & 2251.556 \\
\hline 40 & B4 & 2719.496 & 2297.976 & 2297.976 & 2304.026 & 2253.568 & 2251.550 \\
\hline
\end{tabular}

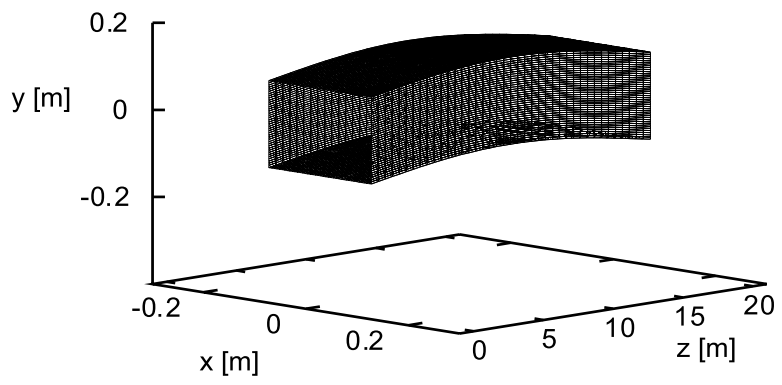

(a) I mode, $\mathrm{f}_{1}=1.195 \mathrm{~Hz}$.

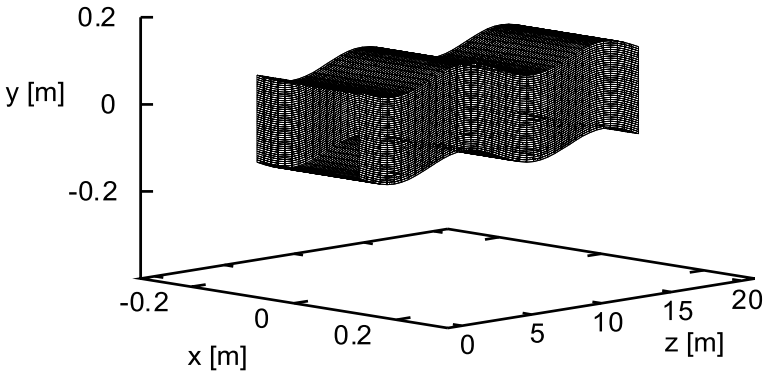

(b) IV mode, $\mathrm{f}_{4}=19.064 \mathrm{~Hz}$.

Fig. 2. Bending modes of the beam with square cross-section. Type of element: B4. Fourth order model. 40 beam elements. $L / h=100$. 
Table 6

Comparison between NASTRAN shell and present beam elements solution on bending and radial natural frequencies, $[\mathrm{Hz}]$. Clamped annular cross-section beam. $L / d=10$

\begin{tabular}{lrc}
\hline & $f_{1}$ & \multicolumn{1}{c}{$f_{2}$} \\
\hline \multicolumn{4}{c}{ Bending Modes } \\
NASTRAN Shell & 5.073 & 29.072 \\
Present Beam, $N=4$ & 5.080 & 29.090 \\
\multicolumn{4}{c}{ Radial Modes } \\
NASTRAN Shell & 40.377 & 121.120 \\
Present Beam, $N=4$ & 40.254 & 120.762 \\
\hline
\end{tabular}

Table 7

Comparison between analytical shell solution and present beam finite element formulation on bending and circumferential natural frequencies, [Hz]. Simply supported annular cross-section beam. $L / d=10$

\begin{tabular}{ccc}
\hline & Analytical Shell & Present Beam, $N=4$ \\
\hline$f_{1_{b}}$ & 14.021 & 14.019 \\
$f_{1_{c}}$ & 22.880 & 23.386 \\
$f_{2_{b}}$ & 51.504 & 51.470 \\
\hline
\end{tabular}

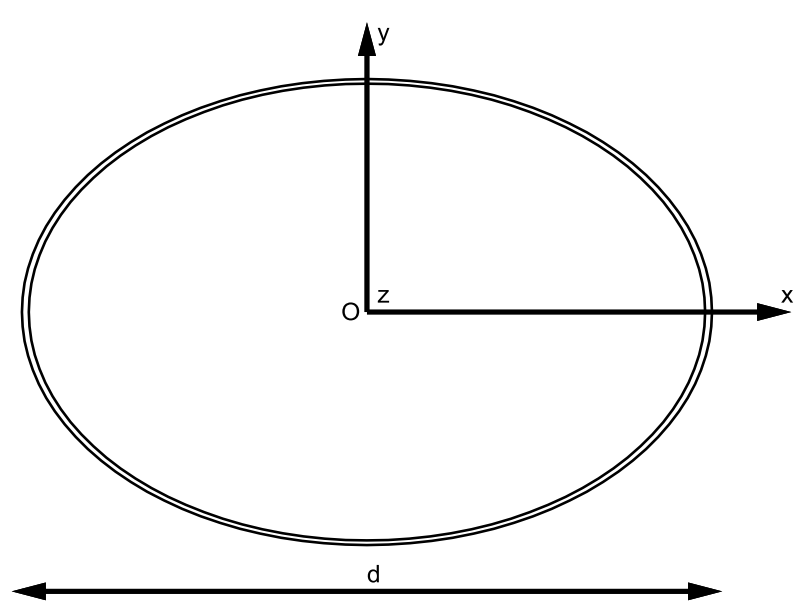

Fig. 3. Coordinate frame and geometry of an annular cross-section.

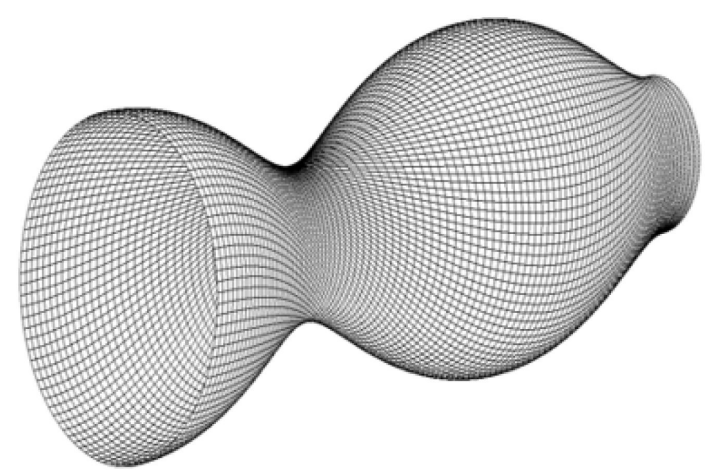

Fig. 4. II radial vibration mode of the annular cross-section beam obtained through NASTRAN shell model. $L / d=5$. 
Table 8

First five bending frequencies, $[\mathrm{Hz}]$, of the beam with annular cross-section. Type of element: B4. 40 beam elements

\begin{tabular}{|c|c|c|c|c|c|}
\hline & $f_{1}$ & $f_{2}$ & $f_{3}$ & $f_{4}$ & $f_{5}$ \\
\hline \multicolumn{6}{|c|}{$L / d=100$} \\
\hline Ref. Sol. Eq. 28 & 0.145 & 0.580 & 1.304 & 2.318 & 3.622 \\
\hline EBBM & $0.145^{(1,2)^{*}}$ & $0.579^{(3,4)}$ & $1.303^{(5,6)}$ & $2.316^{(7,8)}$ & $3.617^{(9,10)}$ \\
\hline TBM & $0.145^{(1,2)}$ & $0.579^{(3,4)}$ & $1.302^{(5,6)}$ & $2.310^{(7,8)}$ & $3.602^{(9,10)}$ \\
\hline$N=1$ & $0.145^{(1,2)}$ & $0.579^{(3,4)}$ & $1.302^{(5,6)}$ & $2.310^{(7,8)}$ & $3.602^{(9,10)}$ \\
\hline$N=2$ & $0.145^{(1,2)}$ & $0.579^{(3,4)}$ & $1.302^{(5,6)}$ & $2.310^{(7,8)}$ & $3.603^{(9,10)}$ \\
\hline$N=3$ & $0.145^{(1,2)}$ & $0.579^{(3,4)}$ & $1.300^{(5,6)}$ & $2.306^{(7,8)}$ & $3.592^{(9,10)}$ \\
\hline$N=4$ & $0.145^{(2,3)}$ & $0.579^{(4,5)}$ & $1.300^{(6,7)}$ & $2.306^{(8,9)}$ & $3.592^{(10,11)}$ \\
\hline \multicolumn{6}{|c|}{$L / d=10$} \\
\hline Ref. Sol. Eq. 28 & 14.489 & 57.958 & 130.405 & 231.831 & 362.235 \\
\hline EBBM & $14.402^{(1,2)}$ & $56.602^{(3,4)}$ & $123.825^{(6,7)}$ & $212.170^{(9,10)}$ & $317.343^{(11,12)}$ \\
\hline TBM & $14.180^{(1,2)}$ & $53.523^{(3,4)}$ & $111.116^{(6,7)}$ & $180.425^{(8,9)}$ & $256.845^{(11,12)}$ \\
\hline$N=1$ & $14.180^{(1,2)}$ & $53.523^{(4,5)}$ & $111.116^{(7,8)}$ & $180.425^{(11,12)}$ & $256.845^{(15,16)}$ \\
\hline$N=2$ & $14.183^{(2,3)}$ & $53.564^{(4,5)}$ & $111.268^{(7,8)}$ & $180.761^{(11,12)}$ & $257.412^{(14,15)}$ \\
\hline$N=3$ & $14.019^{(2,3)}$ & $51.485^{(6,7)}$ & $103.487^{(13,14)}$ & $162.839^{(19,20)}$ & $225.148^{(23,24)}$ \\
\hline$N=4$ & $14.019^{(2,3)}$ & $51.470^{(10,11)}$ & $103.369^{(21,22)}$ & $162.382^{(29,30)}$ & $223.927^{(39,40)}$ \\
\hline
\end{tabular}

$(*)$ : between brackets the positions of the frequencies in the eigenvalues vector are reported.

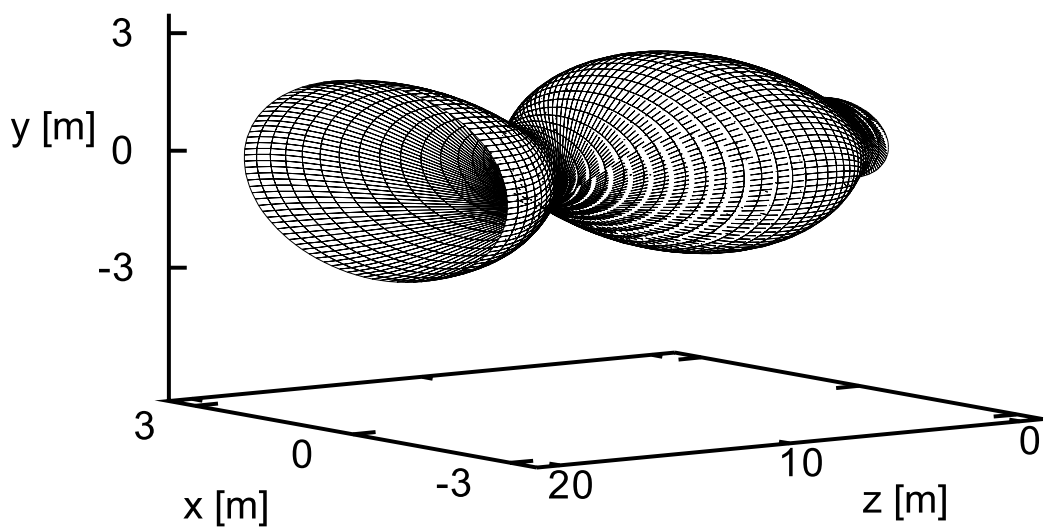

Fig. 5. II radial vibration mode of the annular cross-section beam obtained through the present beam formulation. $N=4 . L / d=5$.

frequency in the eigenvalues vector are reported as well. Each frequency appears twice because of the symmetry of the structure with respect to the longitudinal axis. In case of slender beams, the results match the benchmark values. Considering short beams, higher-order models lead to results which are significantly different from the solutions provided by classical models. Higher the mode number, larger the difference. The usage of refined models permits the identification of a larger amount of vibration modes. The number of modes not predicted by classical models increases as the span-to-height ratio decreases and the expansion order increases. In Fig. 6 the positions of the first three bending frequencies in the eigenvalues vector are plotted for different beam models in case of short beams. With classical theories these frequencies held the first three positions of the vector. New vibration modes appears as the model is refined. In particular, longitudinal and circumferential modes have been found in between the bending ones. In Table 9 the first ten natural frequencies are reported with the indication of the type of vibration mode to whom they are associated with. Short beams are considered. Classical theories are only able to gather bending and longitudinal modes. First- and second-order models can get torsional modes. At least a third-order model is needed to evaluate circumferential modes. Figures 7, 8, 9 and 10 show two circumferential modes. Third-order models furnish two-lobes modes, while three-lobes modes are detected by fourth-order models. In both cases out-of-plane components are observed. The 3-D plots highlight the distribution of the circumferential mode along $z$. 
Table 9

First ten frequencies, $[\mathrm{Hz}]$, of the beam with annular cross-section. Type of element: B4. 40 beam elements. $L / d=10$

\begin{tabular}{ccccccc}
\hline & EBBM & TBM & $N=1$ & $N=2$ & $N=3$ & $N=4$ \\
\hline 1 & $14.402^{b^{*}}$ & $14.180^{b}$ & $14.180^{b}$ & $12.920^{l}$ & $10.973^{l}$ & $0.385^{l}$ \\
2 & $14.402^{b}$ & $14.180^{b}$ & $14.180^{b}$ & $14.183^{b}$ & $14.019^{b}$ & $14.019^{b}$ \\
3 & $56.602^{b}$ & $53.523^{b}$ & $47.888^{l}$ & $14.183^{b}$ & $14.019^{b}$ & $14.019^{b}$ \\
4 & $56.602^{b}$ & $53.523^{b}$ & $53.523^{b}$ & $53.564^{b}$ & $34.798^{c}$ & $23.386^{c}$ \\
5 & $65.881^{l}$ & $65.881^{l}$ & $53.523^{b}$ & $53.564^{b}$ & $34.851^{c}$ & $23.464^{c}$ \\
6 & $123.825^{b}$ & $111.116^{b}$ & $80.508^{t}$ & $80.508^{t}$ & $51.485^{b}$ & $29.214^{c}$ \\
7 & $123.825^{b}$ & $111.116^{b}$ & $111.116^{b}$ & $111.268^{b}$ & $51.485^{b}$ & $29.277^{c}$ \\
8 & $197.642^{l}$ & $180.425^{b}$ & $111.116^{b}$ & $111.268^{b}$ & $61.117^{c}$ & $45.405^{c}$ \\
9 & $212.170^{b}$ & $180.425^{b}$ & $143.558^{l}$ & $132.889^{l}$ & $61.146^{c}$ & $45.445^{c}$ \\
10 & $212.170^{b}$ & $197.642^{l}$ & $161.015^{t}$ & $161.015^{t}$ & $80.508^{t}$ & $51.470^{b}$ \\
\hline
\end{tabular}

$(*)$ : " $b$ " stands for bending mode, "l" stands for longitudinal mode, " $t$ " stands for torsional mode, $c$ stands for circumferential mode.

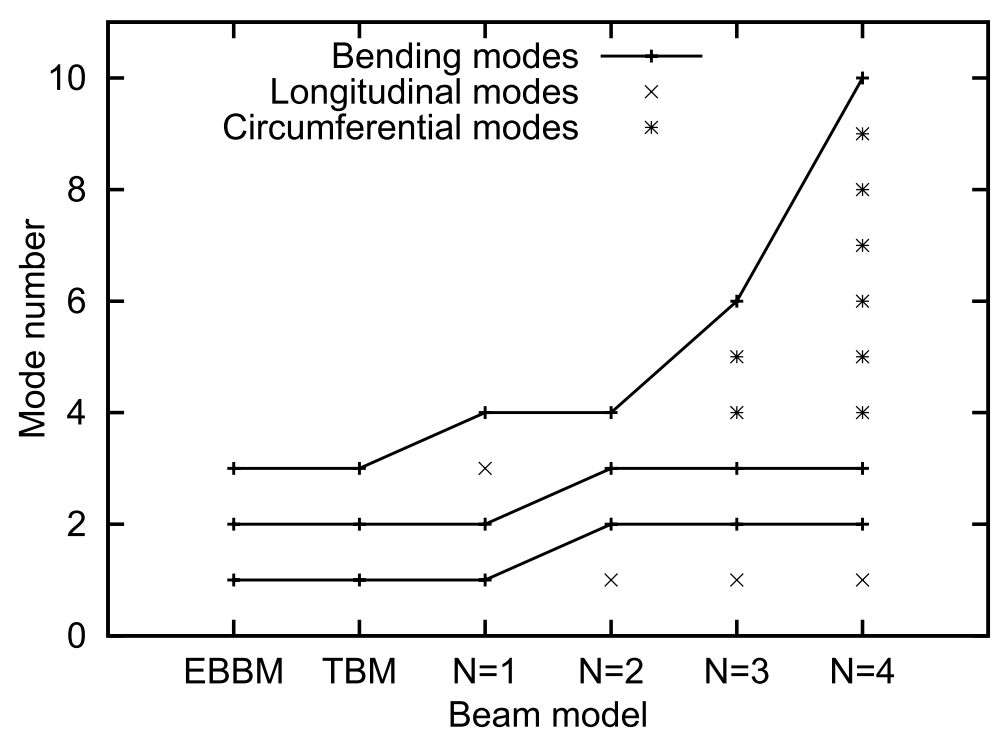

Fig. 6. Frequencies and vibration modes versus beam models in case of annular cross-section. Type of element: B4. 40 beam elements. $L / d=$ 10 .

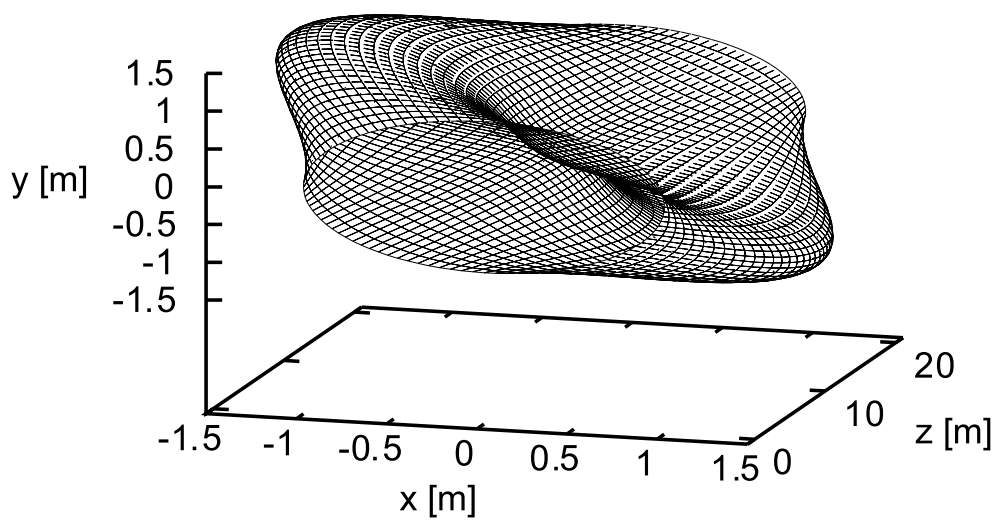

Fig. 7. IV vibration mode of the beam with annular cross-section, $\mathrm{f}_{4}=34.798 \mathrm{~Hz}$. Type of element: B4. Third order model. 40 beam elements. $L / d=10$. 


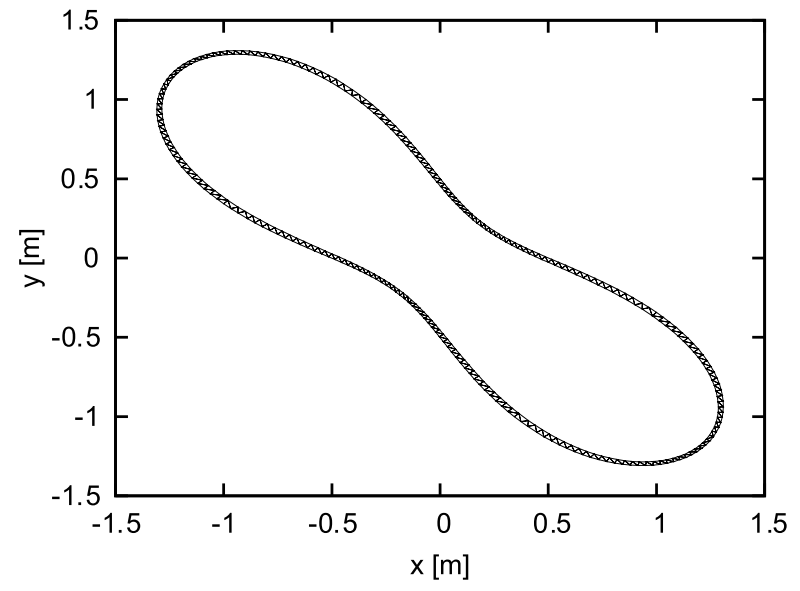

(a) 2-D view.

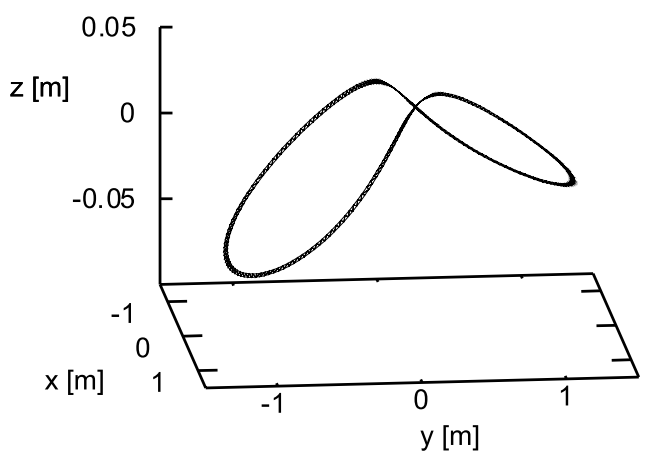

(b) 3-D view.

Fig. 8. IV vibration mode of the annular cross-section at $z=\mathrm{L} / 4, \mathrm{f}_{4}=34.798 \mathrm{~Hz}$. Type of element: B4. Third order model. 40 beam elements. $L / d=10$

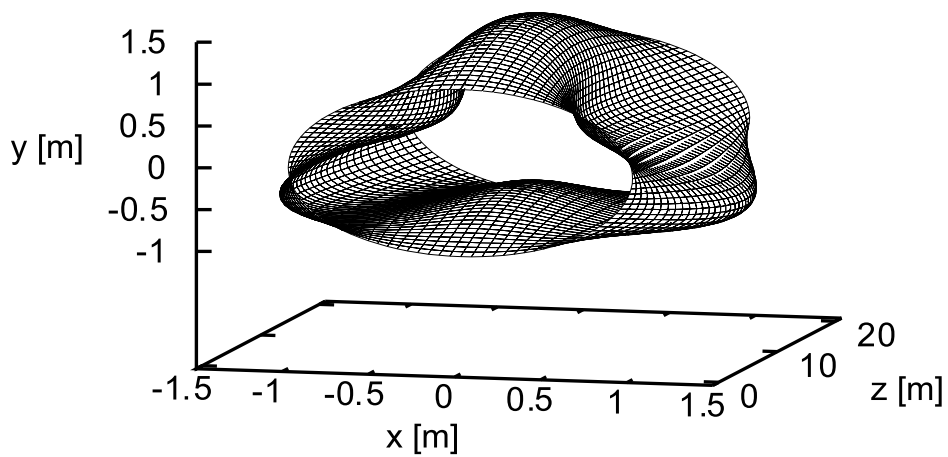

Fig. 9. XV vibration mode of the beam with annular cross-section, $f_{15}=74.204 \mathrm{~Hz}$. Type of element: B4. Fourth order model. 40 beam elements. $L / d=10$

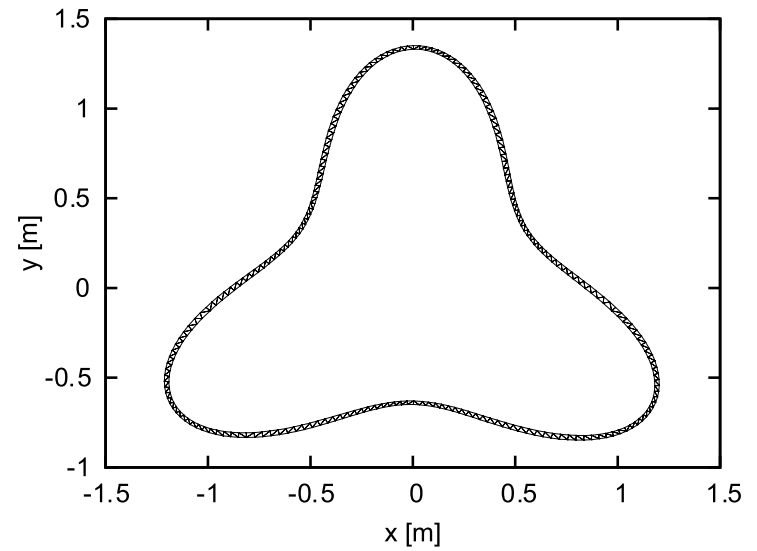

(a) 2-D view.

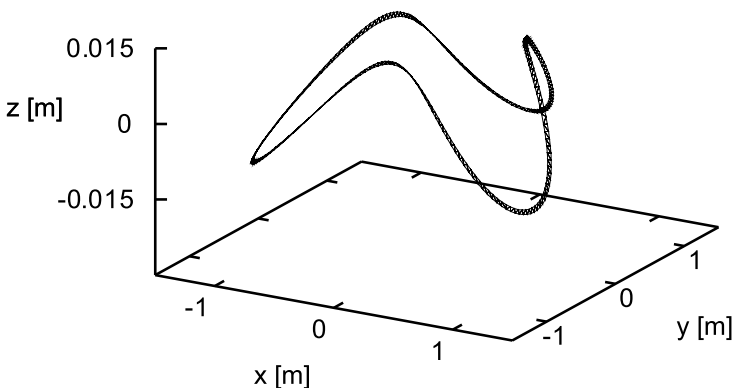

(b) 3-D view.

Fig. 10. XV vibration mode of the annular cross-section at $z=\mathrm{L} / 4, \mathrm{f}_{15}=74.204 \mathrm{~Hz}$. Type of element: B4. Fourth order model. 40 beam elements. $L / d=10$. 
Table 10

First five bending frequencies, $[\mathrm{Hz}]$, of the beam with airfoil cross-section. Type of element: B4. 40 beam elements

\begin{tabular}{|c|c|c|c|c|c|}
\hline & $f_{1}$ & $f_{2}$ & $f_{3}$ & $f_{4}$ & $f_{5}$ \\
\hline \multicolumn{6}{|c|}{$L / b=25$} \\
\hline Ref. Sol. Eq. (29) & 0.235 & 1.472 & 4.122 & 8.078 & 13.352 \\
\hline EBBM & $0.235^{(1)^{*}}$ & $1.470^{(3)}$ & $4.116^{(4)}$ & $8.064^{(5)}$ & $13.328^{(7)}$ \\
\hline TBM & $0.235^{(1)}$ & $1.470^{(3)}$ & $4.114^{(4)}$ & $8.058^{(5)}$ & $13.312^{(7)}$ \\
\hline$N=1$ & $0.235^{(1)}$ & $1.470^{(3)}$ & $4.114^{(4)}$ & $8.058^{(5)}$ & $13.312^{(7)}$ \\
\hline$N=2$ & $0.236^{(1)}$ & $1.476^{(3)}$ & $4.133^{(4)}$ & $8.099^{(5)}$ & $13.388^{(8)}$ \\
\hline$N=3$ & $0.235^{(1)}$ & $1.473^{(3)}$ & $4.119^{(4)}$ & $8.058^{(5)}$ & $13.290^{(8)}$ \\
\hline$N=4$ & $0.235^{(1)}$ & $1.473^{(3)}$ & $4.117^{(4)}$ & $8.052^{(5)}$ & $13.277^{(8)}$ \\
\hline \multicolumn{6}{|c|}{$L / b=5$} \\
\hline Ref. Sol. Eq. (29) & 5.873 & 36.804 & 103.052 & 201.955 & 333.810 \\
\hline EBBM & $5.863^{(1)}$ & $36.692^{(3)}$ & $102.511^{(4)}$ & $200.236^{(6)}$ & $329.634^{(8)}$ \\
\hline TBM & $5.859^{(1)}$ & $36.536^{(3)}$ & $101.491^{(4)}$ & $196.639^{(6)}$ & $320.461^{(8)}$ \\
\hline$N=1$ & $5.859^{(1)}$ & $36.536^{(3)}$ & $101.491^{(4)}$ & $196.639^{(8)}$ & $320.461^{(9)}$ \\
\hline$N=2$ & $5.963^{(1)}$ & $37.071^{(3)}$ & $103.047^{(5)}$ & $199.759^{(8)}$ & $325.149^{(11)}$ \\
\hline$N=3$ & $5.913^{(1)}$ & $36.312^{(3)}$ & $98.705^{(5)}$ & $185.376^{(8)}$ & $290.665^{(11)}$ \\
\hline$N=4$ & $5.903^{(1)}$ & $36.142^{(3)}$ & $97.876^{(5)}$ & $182.813^{(8)}$ & $284.400^{(11)}$ \\
\hline
\end{tabular}

$(*)$ : between brackets the positions of the frequencies in the eigenvalues vector are reported.

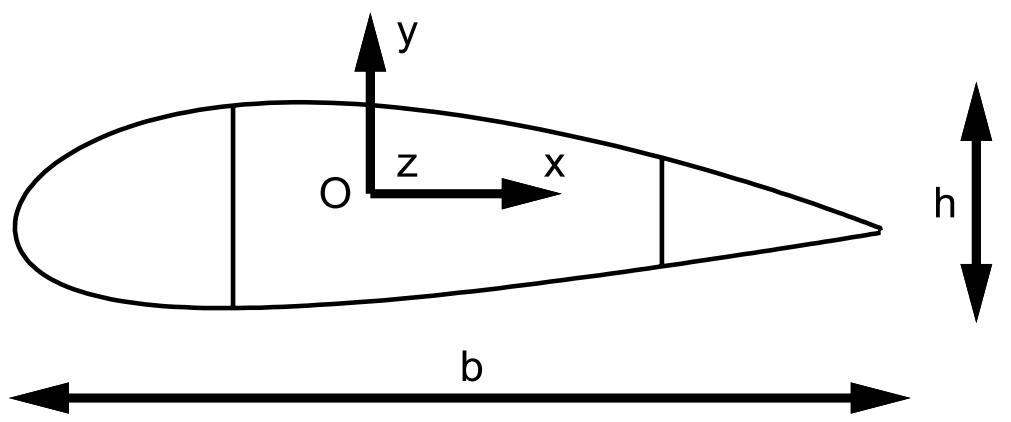

Fig. 11. Coordinate frame and geometry of an airfoil cross-section.

\subsection{Wing and rotor blade cross-sections}

NACA 2415 airfoil profile is adopted as the cross-section, see Fig. 11. The chord length, $b$, is assumed equal to $1[\mathrm{~m}]$. A three cells section is evaluated. The cells are obtained by inserting two beams along the span-wise direction at $25 \%$ and $75 \%$ of the chord. The span-to-chord ratio, $L / b$, is considered as free parameter and is assumed to be equal to 25 and 5 . The first value is characteristic of helicopters rotor blades, while the latter represents a typical value for aircrafts wings. The beam is clamped at $z=0$. Reference values of the $k$-th bending natural frequencies are given by the following expression based on Euler-Bernoulli's assumptions:

$$
f_{k_{b}}=\frac{1}{2 \pi}\left(\frac{\left(\lambda_{k} L\right)^{2}}{L^{2}}\left(\frac{E I}{\rho A}\right)^{\frac{1}{2}}\right.
$$

for the sake of brevity, the values of $\lambda_{k} L$ are not reported here. They can be found in Craig [24].

Table 10 shows the first five natural frequencies related to bending modes along $y$. In case of slender beams, the results match the benchmark values. For short beams, the contribution of the higher-order terms is significant. As far as the position of the bending frequencies in the eigenvalues vector is concerned, refined theories are able to gather more vibration modes than the classical ones. In Table 11 a comparison between the TBM and linear model is conducted by reporting the first ten frequencies and their own mode. Short beams are considered. Timoshenko's model is not able to get the torsional mode since its kinematics assumptions are based on constant distribution of $u_{x}$ and $u_{y}$ above the cross-section. This aspect is also shown in Fig. 12. A longitudinal mode is presented in case 
Table 11

Comparison of the first ten frequencies,

$[\mathrm{Hz}]$, of the beam with airfoil crosssection using TBM and linear expansion. Type of element: B4. 40 beam elements. $L / b=5$

\begin{tabular}{ccc}
\hline & TBM & $N=1$ \\
\hline 1 & $5.859^{b_{y}^{*}}$ & $5.859^{b_{y}}$ \\
2 & $32.692^{b_{x}}$ & $32.692^{b_{x}}$ \\
3 & $36.536^{b_{y}}$ & $36.536^{b_{y}}$ \\
4 & $101.491^{b_{y}}$ & $101.491^{b_{y}}$ \\
5 & $179.031^{b_{x}}$ & $161.015^{t}$ \\
6 & $196.639^{b_{y}}$ & $179.031^{b_{x}}$ \\
7 & $263.523^{l}$ & $191.366^{l}$ \\
8 & $320.461^{b_{y}}$ & $196.639^{b_{y}}$ \\
9 & $431.618^{b_{x}}$ & $320.461^{b_{y}}$ \\
10 & $470.721^{b_{y}}$ & $431.618^{b_{x}}$ \\
\hline
\end{tabular}

$(*):$ " $b_{y}$ " stands for bending mode along $y$;

" $b_{x}$ " stands for bending mode along $x$;

" $l$ " stands for longitudinal mode,

" $t$ " stands for torsional mode.

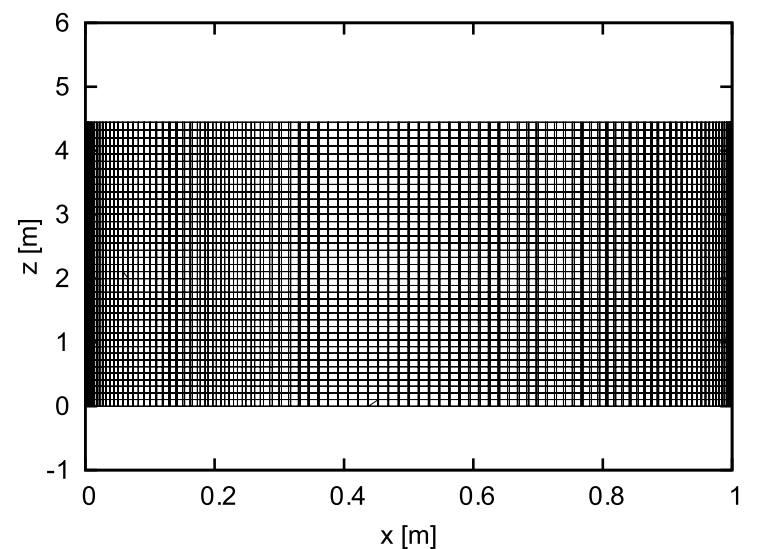

(a) $\mathrm{TBM}, \mathrm{f}_{7}=263.523 \mathrm{~Hz}$.

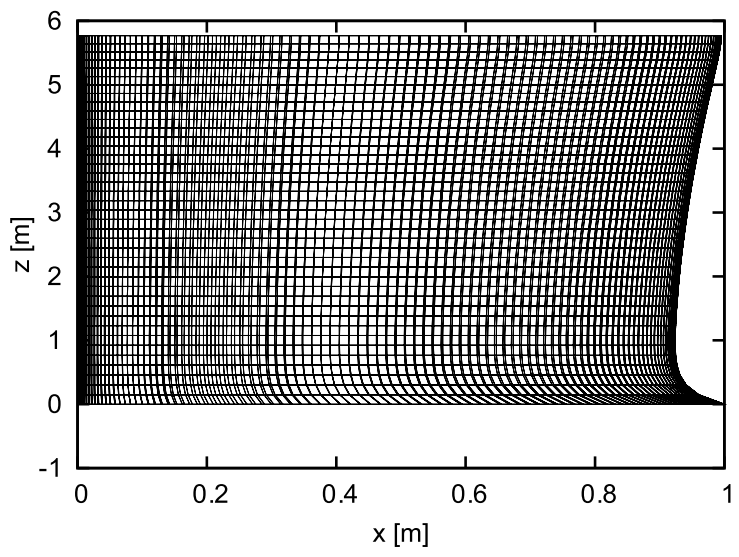

(b) $\mathrm{N}=1, \mathrm{f}_{7}=191.366 \mathrm{~Hz}$.

Fig. 12. VII vibration mode of the airfoil cross-section beam. Comparison between TBM and linear expansion. Type of element: B4. 40 beam elements. $L / b=5$.

of short beams. It has been computed both with the TBM and linear model. In the latter case there are significant contributions of the linear components of $u_{x}$ and $u_{y}$ on the longitudinal mode. The cross-sections undergo in-plane components of displacement which are not constant as $x, y$ and $z$ vary. In Figs 13, 14 and 15 three vibration modes are presented. The adopted model is able to predict the 3-D shape variations of the wing cross-section. In Figs 16 the vibration modes of a section placed at $z=3 \mathrm{~L} / 4$ are shown for different expansion orders in case of short beams. More refined the model, more detailed the computed solution. Higher than second-order models are needed to predict properly the cross-section free vibrations behavior.

\section{Conclusions}

In this paper, free vibrations analysis of beam models based on higher-order theories has been presented. Carrera's Unified Formulation (CUF) has been used for the systemic implementation of refined models. Two-, three- and four-node finite elements have been derived according to CUF. The element stiffness and mass matrices are obtained 


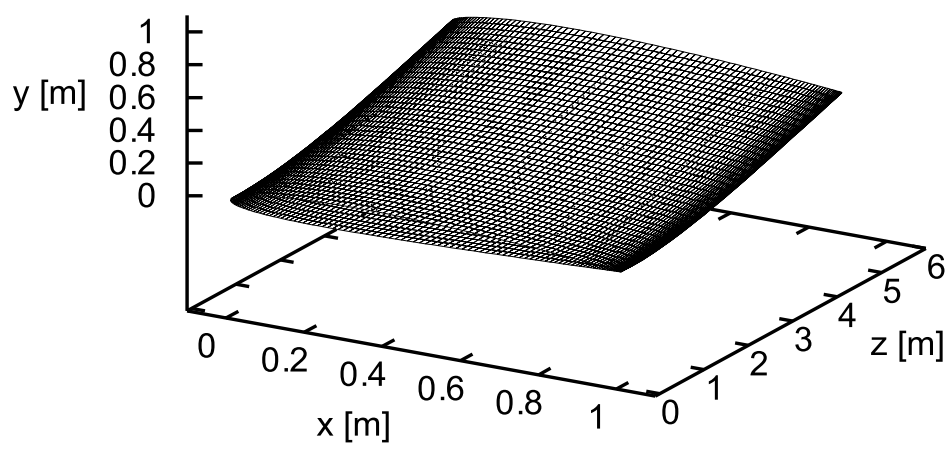

Fig. 13. I vibration mode of the airfoil cross-section beam, $\mathrm{f}_{1}=5.903 \mathrm{~Hz}$. Type of element: $\mathrm{B} 4.40$ beam elements. Fourth order model. $L / b=$ 5 .

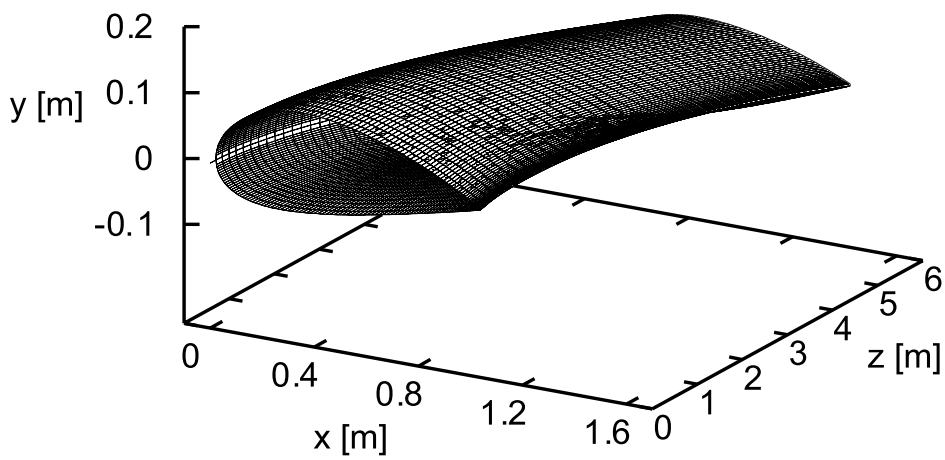

Fig. 14. II vibration mode of the airfoil cross-section beam, $\mathrm{f}_{2}=36.142 \mathrm{~Hz}$. Type of element: B4. 40 beam elements. Fourth order model. $L / b=5$.

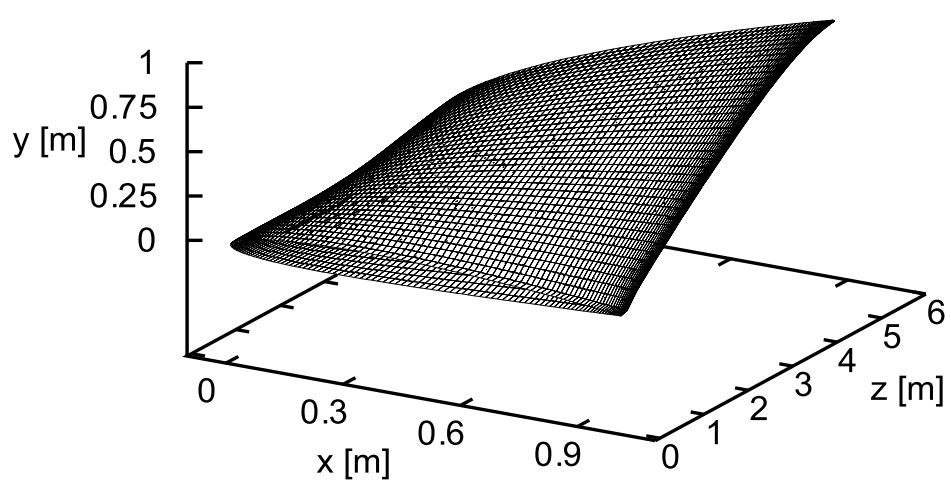

Fig. 15. IV vibration mode of the airfoil cross-section beam, $\mathrm{f}_{4}=182.813 \mathrm{~Hz}$. Type of element: B4. 40 beam elements. Fourth order model. $L / b=5$.

in a compact form, named fundamental nucleus, that does not depend on the theory approximation order. It is assumed as a free parameter of the modeling. It is supposed to be as high as 4 . Elements based on higher-order beam models as well as classical theories can be easily derived. Square, annular and airfoil shaped cross-sections have been accounted for. Slender as well as short beams have been analyzed. Clamped and simply supported boundary conditions have been considered. The dynamics behavior of the beam is described in terms of natural frequencies and vibration modes. Convergency analysis due to the mesh quality has been presented in case of square cross-section through the analysis of the first five natural frequencies. It has been found that it depends on the expansion orders 


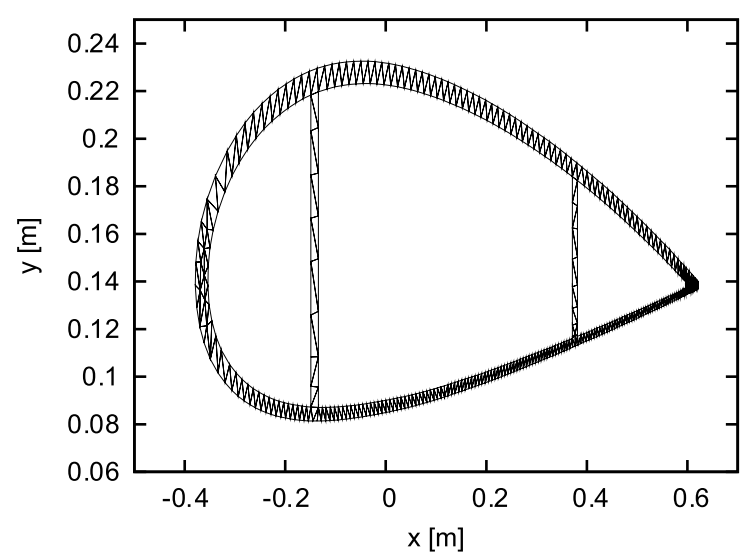

(a) $\mathrm{N}=1, \mathrm{f}_{8}=196.639 \mathrm{~Hz}$.

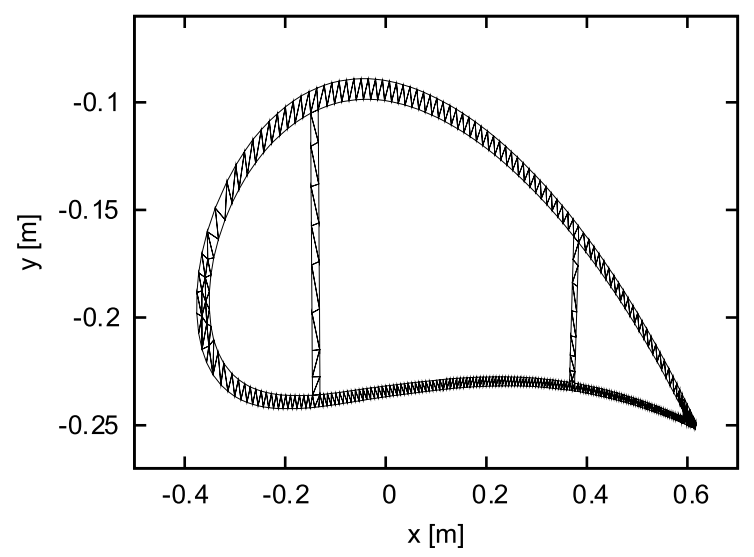

(c) $\mathrm{N}=3, \mathrm{f}_{8}=185.376 \mathrm{~Hz}$.

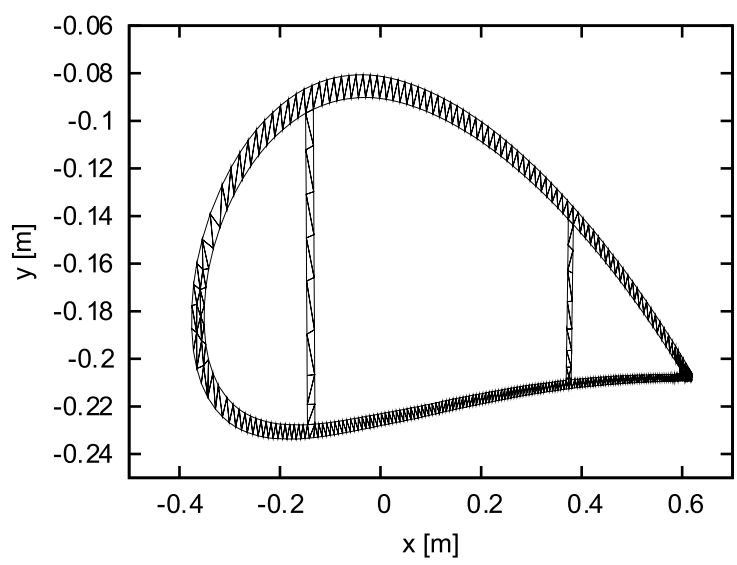

(b) $\mathrm{N}=2, \mathrm{f}_{8}=199.759 \mathrm{~Hz}$

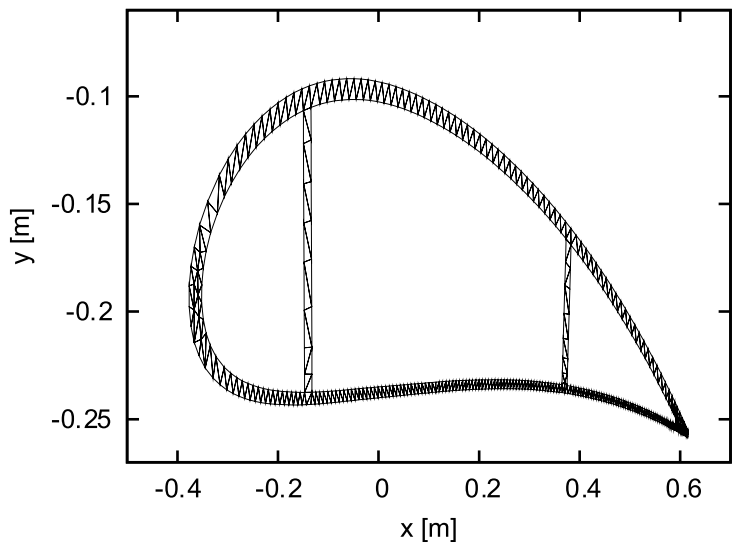

(d) $\mathrm{N}=4, \mathrm{f}_{8}=182.813 \mathrm{~Hz}$.

Fig. 16. VIII vibration mode of the airfoil cross-section at $z=3 \mathrm{~L} / 4$. Type of element: B4. 40 beam elements. $L / b=5$.

especially in case of short beams and higher mode numbers. The analysis of the annular cross-section beam has shown the role of refined models in properly detecting vibration modes characterized by 3 -D features. The wing and rotor blade models have highlighted the differences between different theories. In particular a comparison between Timoshenko's and linear models in predicting longitudinal and torsional vibration modes has been done. It has been shown the capabilities of CUF of accounting for unconventional cross-sections. A refined beam model is necessary to:

- improve the flexibility features of a finite element model;

- obtain accurate values of natural frequencies;

- evaluate 3-D effects on the vibration modes;

- predict shell-type vibration modes in case of thin walled beam sections;

and in particular when:

- unconventional cross-section geometries are adopted;

- short beams are considered;

- higher modes number are investigated;

- the aim of the structural dynamics analysis is the proper prediction of vibration modes such as the torsional or the circumferential ones.

These aspects make the usage of higher-order beam models attractive for aeroeleatic problems where the effectiveness in dealing with modes like the torsional ones is fundamental. The possibility of considering the approximation order 
as a free parameter offered by CUF, makes this formulation particularly suitable for these purposes. Future studies will address the usage of Lagrangian polynomials as expansion functions, application to aeroelastic problems and the implementation of non-linear analysis.

\section{References}

[1] K. Kapania and S. Raciti, Recent advances in analysis of laminated beams and plates, part I: Shear effects and buckling, AIAA Journal 27(7) (1989), 923-935.

[2] K. Kapania and S. Raciti, Recent advances in analysis of laminated beams and plates, part II: Vibrations and wave propagation, AIAA Journal 27(7) (1989), 935-946.

[3] M. Eisenberger, H. Abramovich and O. Shulepov, Dynamic stiffness analysis of laminated beams using a first order shear deformation theory, Composite Structures 31 (1995), 265-271.

[4] S.R. Marur and T. Kant, Free vibration analysis of fiber reinforced composite beams using higher order theories and finite element modelling, Journal of Sound and Vibration 194(3) (1996), 337-351.

[5] S.R. Marur and T. Kant, On the angle ply higher order beam vibrations, Computational Mechanics 40 (2007), $25-33$.

[6] T. Kant, S.R. Marur and G.S. Rao, Analytical solution to the dynamic analysis of laminated beams using higher order refined theory, Composite Structures 40(1) (1998), 1-9.

[7] G. Shi and K.Y. Lam, Finite element vibration analysis of composite beams based on higher-order beam theory, Journal of Sound and Vibration 219(4) (1999), 707-721.

[8] D. Zhou and Y.K. Cheung, The free vibration of a type of tapered beam, Comput Methods Appl Mech Engrg 188 (2000), $203-216$.

[9] M. Kameswara Rao, Y.M. Desai and M.R. Chitnis, Free vibrations of laminated beams using mixed theory, Composite Structures 52 (2001), 149-160.

[10] S.Y. Lee and S.C. Wooh, Finite element vibration analysis of composite box structures using the high order plate theory, Journal of Sound and Vibration 277 (2004), 801-814.

[11] R. Ganesan and A. Zabihollah, Vibration analysis of tapered composite beams using a higher-order finite element. Part I: Formulation, Composite Structures 77 (2007), 306-318.

[12] R. Ganesan and A. Zabihollah, Vibration analysis of tapered composite beams using a higher-order finite element. Part II: parametric study, Composite Structures 77 (2007), 319-330.

[13] M. Şimşek and T. Kocatürk, Free vibration analysis of beams by using a third-order shear deformation theory, Sadhana 32(3) (June 2007), 167-179.

[14] J.-C. Hsu, H.-Y. Lai and C.K. Chen, Free vibration of non-uniform euler-bernoulli beams with general elastically end constraints using adomian modified decomposition method, Journal of Sound and Vibration 318 (2008), 965-981.

[15] E. Carrera, Theories and finite elements for multilayered plates and shells, Archives of Computational Methods in Engineering 9(2) (2002), 87-140.

[16] E. Carrera, Theories and finite elements for multilayered plates and shells: a unified compact formulation with numerical assessment and benchmarking, Archives of Computational Methods in Engineering 10(3) (2003), 216-296.

[17] E. Carrera, G. Giunta, P. Nali and M. Petrolo, Refined beam elements with arbitrary cross-section geometries, Computers and Structures, DOI: 10.1016/j.compstruc.2009.11.002. Available online

[18] S.W. Tsai, Composites Design, Dayton, Think Composites, 4th edition, 1988.

[19] J.N. Reddy, Mechanics of laminated composite plates and shells. Theory and Analysis, CRC Press, 2nd edition, 2004.

[20] E. Carrera and S. Brischetto, Analysis of thickness locking in classical, refined and mixed multilayered plate theories, Composite Structures 82(4) (2008), 549-562.

[21] E. Carrera and S. Brischetto, Analysis of thickness locking in classical, refined and mixed theories for layered shells, Composite Structures 85(1) (2008), 83-90.

[22] E. Carrera and G. Giunta, Refined beam theories based on Carrera's unified formulation, International Journal of Applied Mechanics (2009), in press.

[23] K.J. Bathe, Finite element procedure, Prentice hall, 1996.

[24] R.R. Craig, Jr., Structural dynamics, John Wiley and Sons, 1981.

[25] E. Carrera, Multilayered shell theories that account for a layer-wise mixed description. Part I: Governing equations, AIAA Journal 37 (1999), 1107-1116.

[26] E. Carrera, Multilayered shell theories that account for a layer-wise mixed description. Part II: Numerical evaluations, AIAA Journal 37 (1999), 1117-1124.

[27] E. Carrera, A study of transverse normal stress effects on vibration of multilayered plates and shells, Journal of Sound and Vibration 225 (1999), 803-829. 

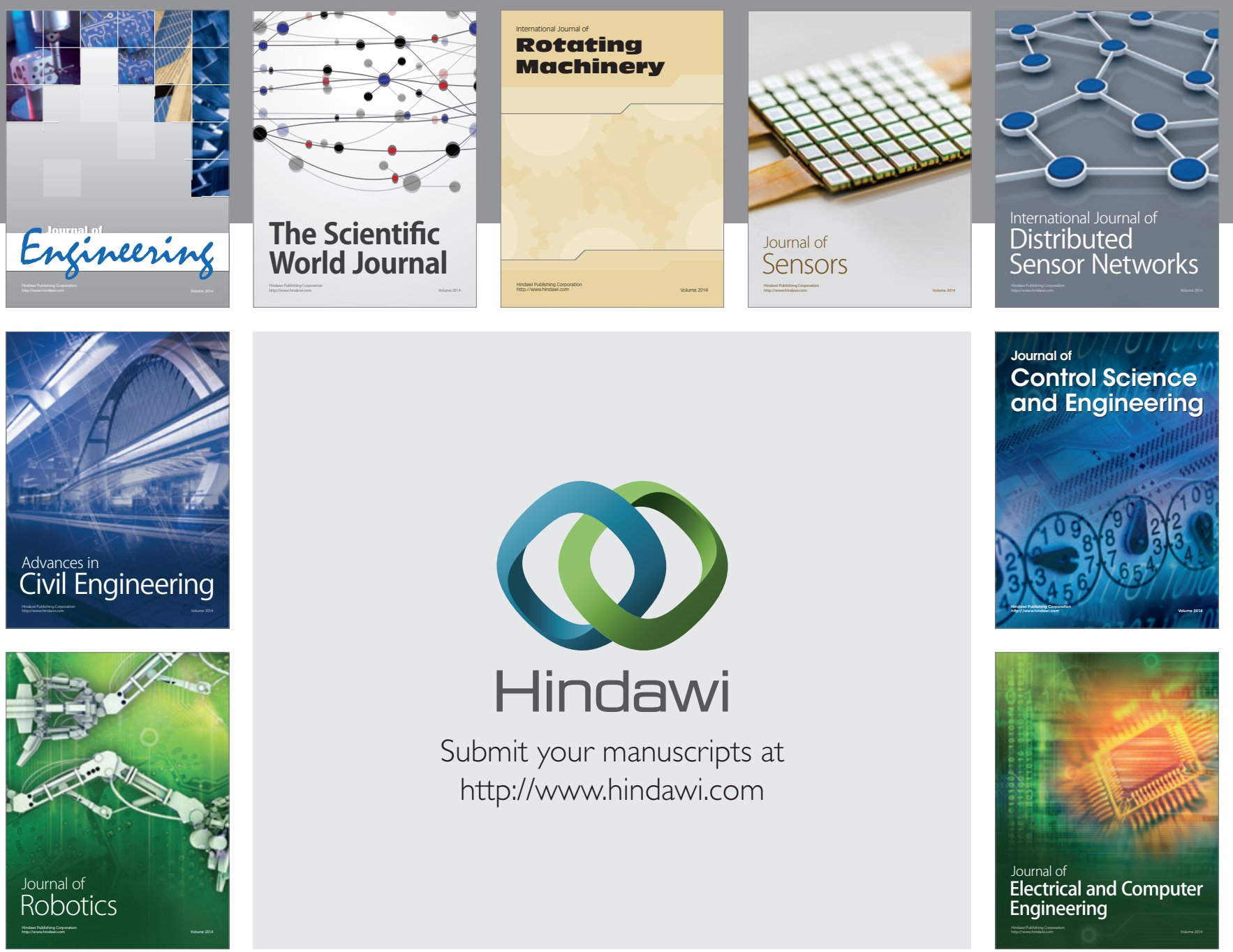

Submit your manuscripts at

http://www.hindawi.com
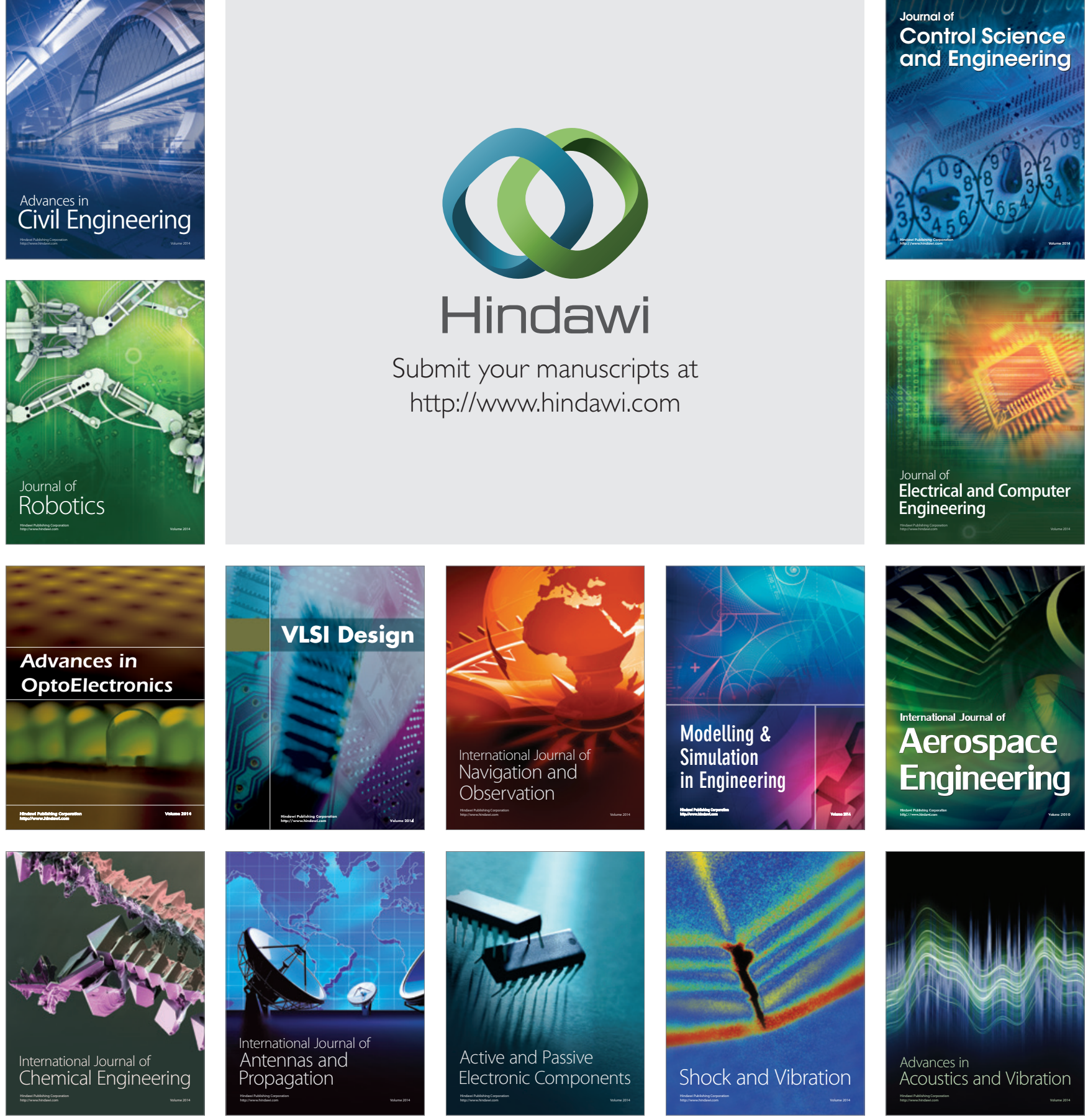